\title{
Osmotic and Electroosmotic Fluid Transport across the Retinal Pigment Epithelium: a Mathematical Model
}

DOI:

10.1016/j.jtbi.2018.08.009

\section{Document Version}

Accepted author manuscript

Link to publication record in Manchester Research Explorer

\section{Citation for published version (APA):}

Dvoriashyna, M., Foss, A. J. E., Gaffney, E. A., Jensen, O., \& Repetto, R. (2018). Osmotic and Electroosmotic Fluid Transport across the Retinal Pigment Epithelium: a Mathematical Model. Journal of Theoretical Biology, 456, 233-248. https://doi.org/10.1016/j.jtbi.2018.08.009

\section{Published in:}

Journal of Theoretical Biology

\section{Citing this paper}

Please note that where the full-text provided on Manchester Research Explorer is the Author Accepted Manuscript or Proof version this may differ from the final Published version. If citing, it is advised that you check and use the publisher's definitive version.

\section{General rights}

Copyright and moral rights for the publications made accessible in the Research Explorer are retained by the authors and/or other copyright owners and it is a condition of accessing publications that users recognise and abide by the legal requirements associated with these rights.

\section{Takedown policy}

If you believe that this document breaches copyright please refer to the University of Manchester's Takedown Procedures [http://man.ac.uk/04Y6Bo] or contact uml.scholarlycommunications@manchester.ac.uk providing relevant details, so we can investigate your claim.

\section{OPEN ACCESS}




\title{
Osmotic and Electroosmotic Fluid Transport across the Retinal Pigment Epithelium: a Mathematical Model
}

\author{
Mariia Dvoriashyna ${ }^{\mathrm{a}, *}$, Alexander J. E. Foss ${ }^{\mathrm{b}}$, Eamonn A. Gaffney ${ }^{\mathrm{c}}$, Oliver E. Jensen ${ }^{\mathrm{d}}$, \\ Rodolfo Repetto ${ }^{\mathrm{a}}$ \\ ${ }^{a}$ Department of Civil, Chemical and Environmental Engineering, University of Genoa, Via Montallegro 1, \\ Genoa, 16145, Italy \\ ${ }^{b}$ Department of Ophthalmology, Nottingham University Hospitals NHS Trust, Nottingham NG5 1PB, UK \\ ${ }^{c}$ Wolfson Centre for Mathematical Biology, Mathematical Institute, University of Oxford, Oxford OX2 6GG, \\ $U K$ \\ ${ }^{d}$ School of Mathematics, University of Manchester, Manchester M13 9PL, UK
}

\begin{abstract}
The retinal pigment epithelium (RPE) is the outermost cell layer of the retina. It has several important physiological functions, among which is removal of excess fluid from the sub-retinal space by pumping it isotonically towards the choroid. Failure of this pumping leads to fluid accumulation, which is closely associated with several pathological conditions, such as age-related macular degeneration, macular oedema and retinal detachment. In the present work we study mechanisms responsible for fluid transport across the RPE with the aim of understanding how fluid accumulation can be prevented. We focus on two possible mechanisms, osmosis and electroosmosis, and develop a spatially resolved mathematical model that couples fluid and ion transport across the epithelium, accounting for the presence of $\mathrm{Na}^{+}, \mathrm{K}^{+}$and $\mathrm{Cl}^{-}$ions. Our model predicts spatial variability of ion concentrations and the electrical potential along the cleft gap between two adjacent cells, which osmotically drives the flow across the lateral membranes. This flow is directed from the sub-retinal space to the choroid and has a magnitude close to measured values. Electroosmosis is subdominant by three orders of magnitude to osmosis and has an opposite direction, suggesting that local osmosis is the main driving mechanism for water transport across the RPE.
\end{abstract}

Keywords: epithelial transport, fluid accumulation in the sub-retinal space,

\footnotetext{
* Corresponding author

Email address: mariia.dvoriashyna@edu.unige.it (Mariia Dvoriashyna)
} 
electroosmosis, local osmosis

\section{Introduction}

The retina is a light-sensitive tissue approximately $0.5 \mathrm{~mm}$ thick, which is located in the posterior part of the eye between the vitreous chamber and the choroid [1]. The retinal pigment epithelium (RPE) is the outermost layer of the retina that separates the photoreceptors from Bruch's membrane and the choroidal blood supply [2]. The RPE is a monolayer of cuboidal epithelial cells, arranged as hexagonal tiles approximately $14 \mu \mathrm{m}$ in diameter in the central retina. The epithelial cells are bound together by junctional complexes with tight junctions, that separate the extracellular regions into apical and basolateral [2] (see Fig 1). The basal side of the RPE faces Bruch's membrane, which is elastic and approximately $2 \mu \mathrm{m}$ thick. The apical membrane borders the sub-retinal space, the extracellular space surrounding the photoreceptor cells [3]. This membrane forms long microvilli (5-7 $\mu \mathrm{m}$ long), that interdigitate the rod outer segments. The main functions of the RPE include creating a barrier between the choroid and retina, phagocytosing shed photoreceptor outer segments and transporting 15 water, ions and metabolites between the retina and the choroid [2]. In particular, a fundamental role of the RPE is the removal of excess fluid from the sub-retinal space [3].

The RPE transports ions, the largest concentrations of which are $\mathrm{Na}^{+}, \mathrm{K}^{+}, \mathrm{Cl}^{-}$and $\mathrm{HCO}_{3}^{-}$. The transepithelial transport of these ions has been studied in vitro in various species and the mechanisms for this transport have been quantitatively identified, e.g. [4, 5, 6]. The route and mechanisms for the transepithelial water transport, on the other hand, are less well understood [7]. The rate of transport has been estimated to range from 1.4 to $11 \mu \mathrm{l} / \mathrm{cm}^{2} / \mathrm{h}$ and is hypothesised to follow a transcellular route, directed from the apical to basolateral side, based on the fact that the RPE is a tight epithelium 25 [8].

Under normal physiological conditions, fluid has to be pumped out of the subretinal space. This process regulates normal retinal adhesion, helps recovery from retinal detachment, and acts as one of the pathways for fluid outflow from the eye [4]. 
Failure of the fluid pump leads to accumulation of fluid in the sub-retinal space, which is closely associated with the formation and development of numerous pathological conditions, such as age-related macular degeneration, diabetic macular oedema and retinal detachment [9]. Hence, quantifying and classifying the mechanisms that drive fluid flow across the RPE is relevant to develop a predictive understanding of how fluid accumulation may be ameliorated, motivating the present work.

RPE is an absorptive epithelium, where the water and salt are transported isotonically (i.e. without significant difference of the solution concentration in the apical and basal sides) [10]. Due to the small geometrical scales, performing experimental observations is challenging, motivating the application of mathematical modelling to understand physiological transport in the RPE. For other epithelia several models have 40 been developed to describe isotonic transepithelial water transport [11, 7, 12, 13, 14], among which the most long-standing driving mechanism is considered to be flow due to local osmosis in the narrow space between cells, induced by concentration gradients in this space [15, 16]. The validity of the theory, however, has been criticised [17, 14], mainly due to the use of questionable geometrical parameters and membrane hydrodynamic permeabilities, which are governed by the presence of aquaporins (AQPs). In particular, it has been shown that knockout of AQPs in different epithelia in mice results in a decrease of osmotic permeability, for example by a factor of between $0.11-0.22$ for the murine proximal tubule, with about a 50\% decrease in water transport [18].

The other mechanism, which has been extensively studied recently in other cell layers, particularly in the corneal endothelium, is electroosmosis [19, 13, 20]. Electroosmosis occurs due to the interaction of an electric field with non-zero net charge located in the Debye layer (i.e. the layer of positively charged species attracted to a negatively charged cell membrane), which results in a Coulomb body force acting on the fluid [21]. The transepithelial electrical potential (TEP) in the RPE has been measured to be around 5-15 $\mathrm{mV}$ (apical positive) [6], which indicates the possibility that the electric field drives an electroosmotic flow. To evaluate the prospect of electroosmotic contributions to water transport in the RPE, and epithelia more generally, a model of ion transport is required that can encompass both osmolarities and the possible generation of electric fields. 


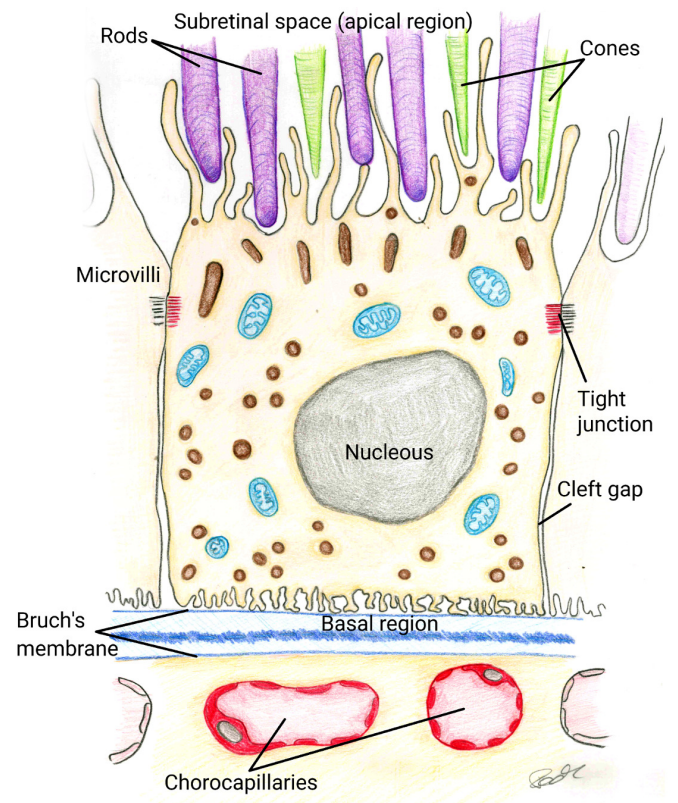

Figure 1: Sketch of the RPE cell layer. Drawing by Federica Grillo.

In the present work we develop an integrated mathematical model based on electrodiffusion that captures both ion and fluid transport in the RPE, together with their coupling, as detailed in Section 2 The first objective is to determine the relative importance of the mechanisms that drive water flow across the RPE with a further, more general, aim of developing a framework for a spatially resolved system to model fluid and ion transport in absorptive epithelia. The choice of the model parameters is described in the Section 3 , the results of the model are reported in Section 4 and the discussion and conclusions of the work are presented in Section 5 Mathematical details of the model are reported in the Appendix.

\section{Methods}

\subsection{Model setup}

We model the RPE cell layer as a two-dimensional row of rectangles with length $L$ and width $H$ as in Fig. 2, separated by a distance $2 h$, which constitutes the cleft gap. As schematically shown in Fig. 1, the apical membrane of the cells is highly 


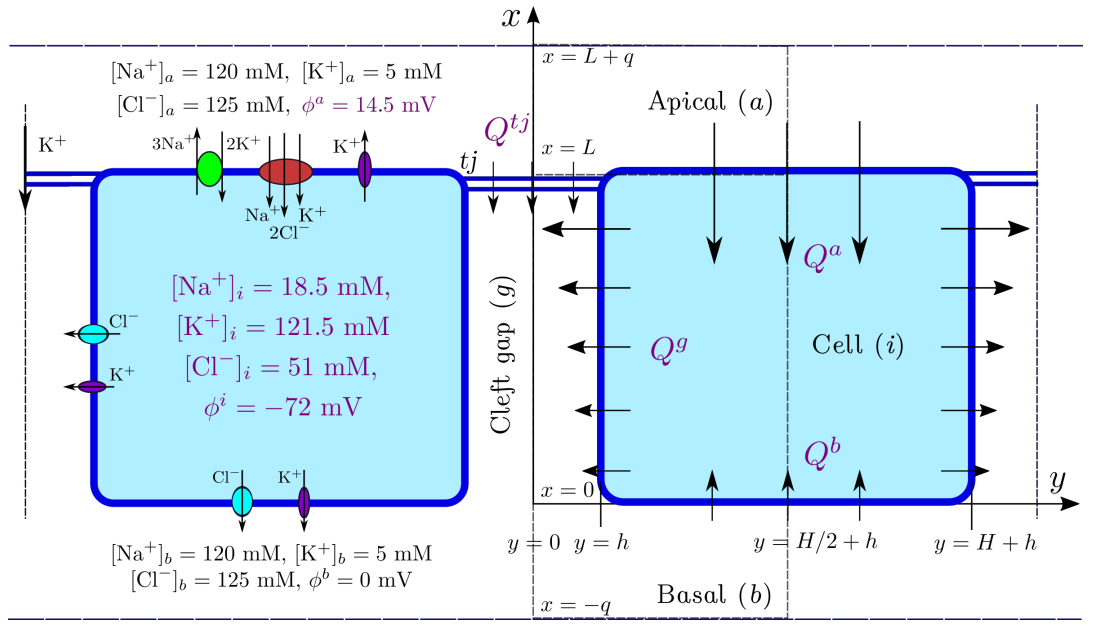

Figure 2: Sketch of the idealised geometry of the RPE. Cells have height $L$, width $H$ and the thickness of the cleft gap is $2 h$. Left panel: transepithelial transport of ions considered in the model and ion concentrations. The apical membrane has $\mathrm{Na}^{+} / \mathrm{K}^{+}$-ATPases (depicted in green), $\mathrm{K}^{+}$channels (purple) and $\mathrm{Na}^{+} / \mathrm{K}^{+} / 2 \mathrm{Cl}^{-}$cotransporters (red). $\mathrm{Cl}^{-}$(light blue) and $\mathrm{K}^{+}$(purple) channels are present in the basolateral membrane. The tight junction is permeable to $\mathrm{K}^{+}$. The values of concentration and electrical potential in the cell and extracellular space are shown. The values computed from the model are $\left[\mathrm{Na}^{+}\right]_{i},\left[\mathrm{~K}^{+}\right]_{i},\left[\mathrm{Cl}^{-}\right]_{i}, \phi^{i}, \phi^{a}$ (reported in purple) and other values are prescribed (in black). Right panel: the coordinate system used in the model and water fluxes directions. The dashed box represents the 'reference' frame. The arrows represent schematically the directions and magnitude of the fluid flow across the membranes. $Q^{s}(s \in\{a, b, g, t j\})$ denotes the flux through the membrane $s . t j$ denotes the tight junction. The sketch is not to scale.

folded, having a surface area approximately 30 times larger than the basolateral one in amphibians [22] and 10 times in mammals [23]. We neglect this folding in the geometrical domain, but consider its effect on the transport properties of the membrane via geometrical adjustments to permeability coefficients for water and ions.

In our model we consider four different regions: apical (or sub-retinal space) (a), intracellular space $(i)$, the basal (or Bruch's membrane) $(b)$ and the cleft gap between two cells (or paracellular space) ( $g$ ) (see Figure 2). Every extracellular region is separated from the cell by a membrane that we name according to the respective region $(a, b, g)$. The apical region is separated from the cleft gap by a tight junction $(t j)$, while the basal one is directly connected to the cleft gap.

We consider that RPE cells are located in a physiological solution of $\mathrm{NaCl}$ and $\mathrm{KCl}$, which dissociate into $\mathrm{Na}^{+}, \mathrm{K}^{+}$and $\mathrm{Cl}^{-}$ions. We neglect the presence of $\mathrm{HCO}_{3}^{-}$ and $\mathrm{H}^{+}$, because $\mathrm{Na}^{+}, \mathrm{K}^{+}$and $\mathrm{Cl}^{-}$represent the major ion fluxes across the RPE [24]. Thus, $\mathrm{Cl}^{-}$is the only anion accounted for in the extracellular space. The cell however, 


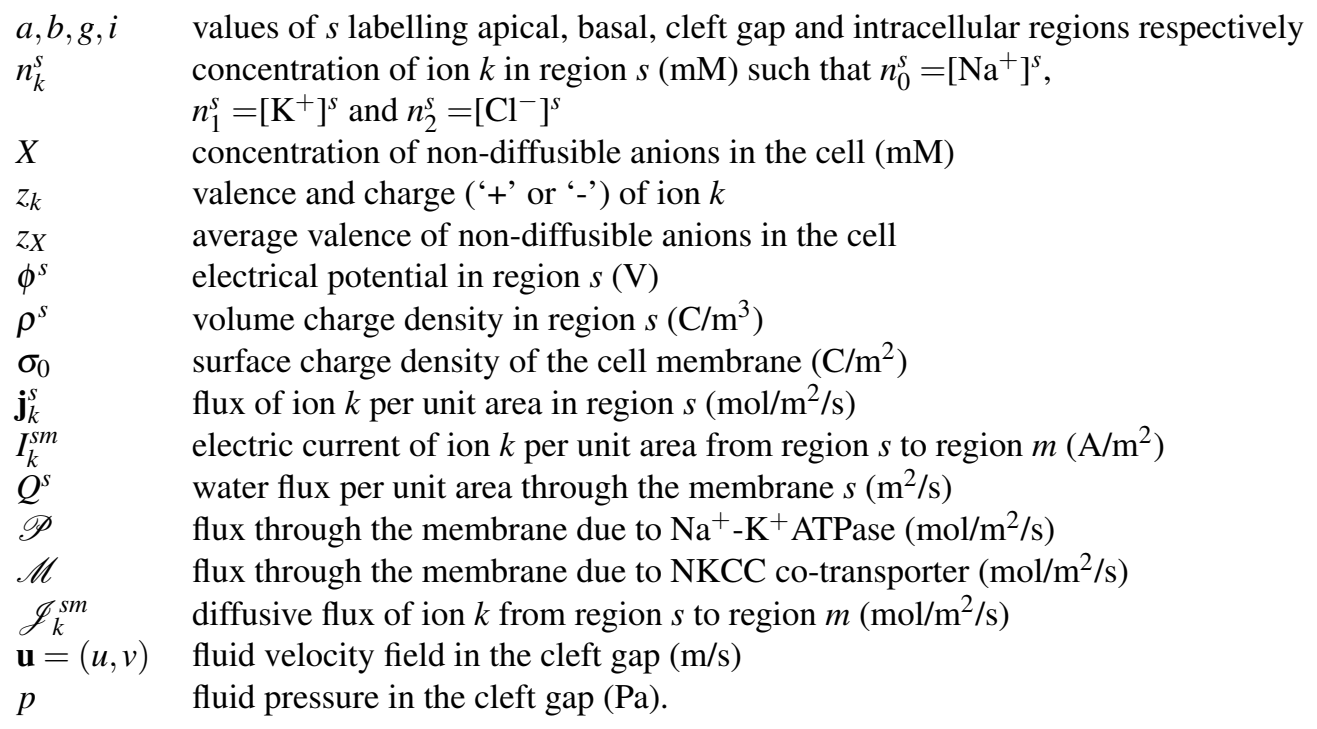

Table 1: A list of parameters and variables used in the model, together with their units.

has an anion gap due to the presence of large non-diffusible molecules, the concentration of which we denote with $X$ and the corresponding fixed negative charge with $X^{i}:=z_{X} X$, where $z_{X}$ is the mean valence of these molecules. We account for active and passive ion transport across the cell membranes. In particular, on the apical membrane we consider the presence of $\mathrm{Na}^{+}-\mathrm{K}^{+}$ATPases (active transport), the channels which cotransport $\mathrm{Na}^{+}, \mathrm{K}^{+}$and two $\mathrm{Cl}^{-}$(the NKCC channel) and $\mathrm{K}^{+}$channels (see Figure 2). The basolateral membrane is assumed to have $\mathrm{K}^{+}$and $\mathrm{Cl}^{-}$channels [25, 6]. The tight junction is considered to be potassium selective [26]. As possible mechanisms that drive water flow across the RPE we consider transcellular osmosis and electroosmosis along the cleft gap.

Throughout the paper we adopt the notation presented in Table 1 1 Unless stated otherwise, we use superscripts to label regions and subscripts to label ions.

The modelling procedure is split into several steps. Although fluid and ion transport are fully coupled, we present the corresponding models individually and simplify them using asymptotic expansions. First we construct the model for ion transport, accounting for the spatial variability of the electrical field and species concentrations. Due to the possibility of electroosmosis, we also need to resolve the Debye layer at the cell 
membrane in the cleft gap and, in particular, predict its surface charge. We therefore develop a solution in the double layer, which is relevant for the fluid flow in the cleft. Then, we model the fluid flow in the cleft. Ion and fluid transport are fully coupled because of the appearance of an advective term in ion fluxes and also because osmotic water fluxes are based on jumps of ion concentration across the membranes.

\subsection{Ion transport}

Since we are interested in studying water transport across the RPE we do not need to consider transient variations of the ion concentrations and the electrical potential, induced for instance by response to variations in light conditions, and look instead for steady-state solutions. The ion transport model in region $s$ consists of the conservation of fluxes for each ion $k$ (Nernst-Plank equations) and the Poisson equation for the electrical potential [21]

$$
\begin{aligned}
& \nabla \cdot \mathbf{j}_{k}^{s}=0, \quad \mathbf{j}_{k}^{s}=-D_{k}\left(\nabla n_{k}^{s}+\frac{z_{k} F}{R T} n_{k}^{s} \nabla \phi^{s}\right)+\mathbf{u} n_{k}^{s}, k \in\{0,1,2\}, \\
& \nabla^{2} \phi^{s}=-\frac{\rho^{s}}{\varepsilon}, \quad \rho^{s}=F\left(\sum z_{k} n_{k}^{s}-X^{s}\right), \quad s \in\{a, b, i, g\},
\end{aligned}
$$

where $X^{i}=X z_{X}>0$ is a fixed negative charge in the cell and is assumed constant, and $X^{s}$ is zero in all other regions $(s \neq i)$. For simplicity, we assume that the diffusion coefficient $D_{k}$ of all considered ions is the same, and is denoted as $D$ below. All other variables and parameters are defined in Table 1 and 2 . It can be verified a posteriori that the Péclet number of the flow is typically small $(P e \approx 0.04)$ and thus it might be tempting to drop advection from ion transport (the term $\mathbf{u} n_{k}^{s}$ in $1 \mathrm{a}$ ). However, the Péclet number might be a slightly misleading estimate of the importance of advection over diffusion when the concentration drop is small with respect to the concentration itself, which is the case in this model, in particular for $\mathrm{Na}^{+}$. Advective terms are found to play non-negligible role and are thus retained in the model. 


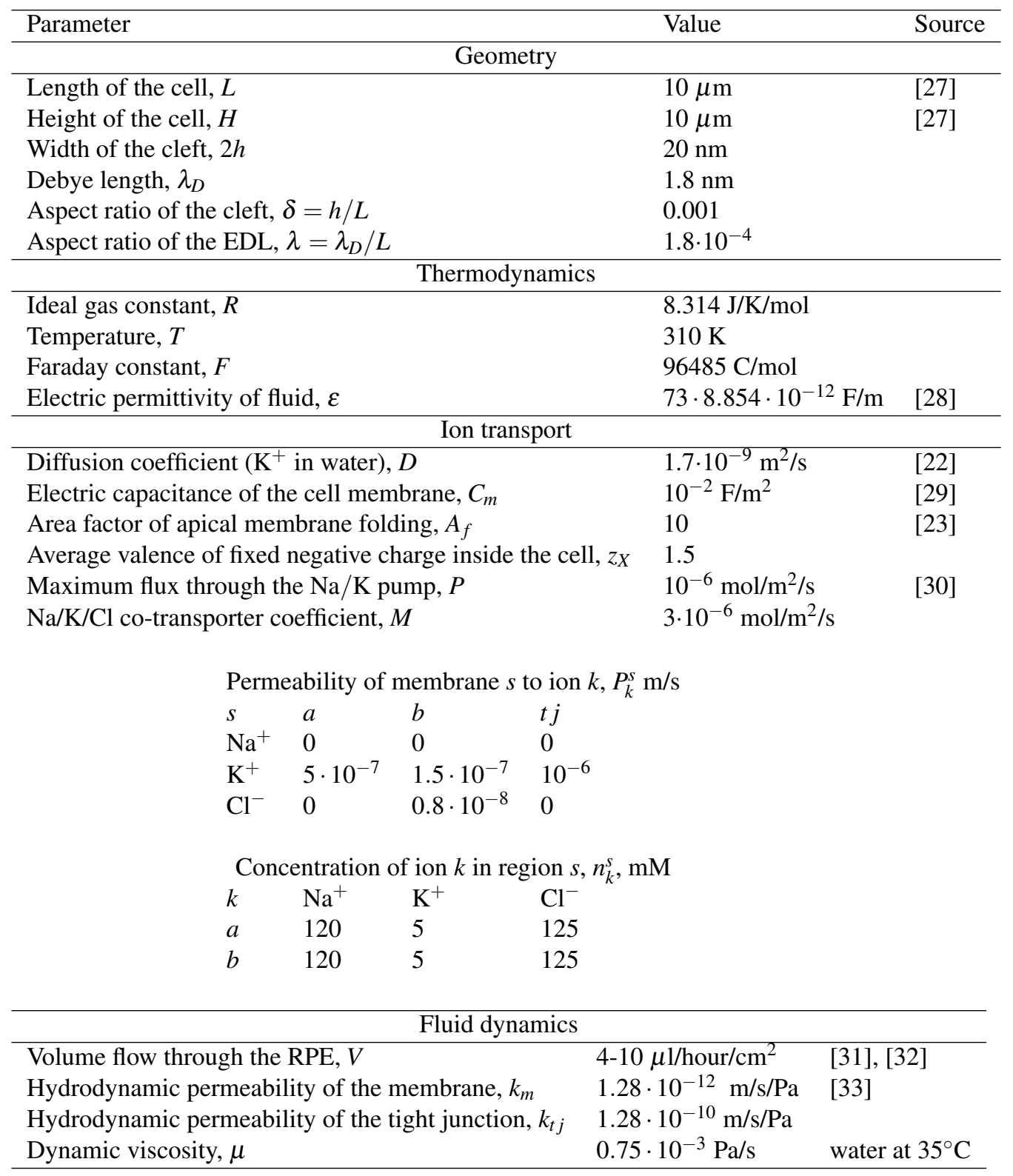

Table 2: Model reference parameters; please refer to the text for further details on their motivation. 


\subsubsection{The bulk}

We assume that the solution in the bulk of each region is electroneutral, which implies

$$
\sum_{k=0,1,2} z_{k} n_{k}^{s}=X^{s}, s \in\{a, b, i, g\} .
$$

This does not need to hold within a few Debye lengths of the cell membranes, which have a lengthscale of nanometres [34, 35]. The use of [2] is justified by the observation that cellular systems will relax to electroneutrality in the bulk on a timescale of nanoseconds [34, 35] and we neglect such fast dynamics.

Eq. (1b) cannot be used together with the electroneutrality assumption [21] as they are not independent. Instead, following the approach of Mori et al. [36, 37], given electroneutrality in the bulk of each region, we can weight the equations (1a) for ion $k$ by $z_{k}$, sum them over the species and use (2) to obtain the following equation for the electrical potential

$$
\nabla \cdot\left(\left[\sum_{k=0}^{2} n_{k}^{s}\right] \nabla \phi^{s}\right)=0 .
$$

We finally eliminate chloride concentration $n_{2}^{s}$ as a variable using (2). Therefore, in the bulk of every region we have three unknowns, $n_{0}^{s}, n_{1}^{s}$ and $\phi^{s}$, and the corresponding two second-order conservation Eqs. (1a) (for $k=0,1)$ and (3) for the electrical potential.

All the membranes and the tight junction are subject to membrane-type conditions. At the membrane between the generic regions $s$ and $m$, we impose the conservation of ion fluxes for each species $k$, which is implemented by imposing that the ion current $I_{k}^{s m}$ on the $s$ side of the membrane equals the flux of charge due to the movement of species $k$ passing from $s$ to $m$,

$$
F z_{k} \mathbf{n}^{s m} \cdot \mathbf{j}_{k}^{s}=I_{k}^{s m}
$$

where $\mathbf{n}^{s m}$ is the unit normal pointing from region $s$ to region $m$. The current $I_{k}^{s m}$ is prescribed to account for all passive and active flows of ion $k$ due to channels and pumps on the corresponding membrane and it is a nonlinear function of concentrations and potential on both sides of the membrane. The choice of constitutive relations for 
these currents is described in Section 2.2.2 Note that the above membrane conditions 'jump' across the Debye layers and involve only the values of the variables in the bulk.

Noting that $\mathrm{Cl}^{-}$concentrations are no longer independent variables due to their elimination via electroneutrality (2), it is convenient to implement a summation over the species, giving a condition in the terms of the potential; in particular rather than the chloride boundary condition we use

$$
\sum_{k} F z_{k} \mathbf{n}^{s m} \cdot \mathbf{j}_{k}^{s}=\sum_{k} I_{k}^{s m}
$$

To close the system, we have Dirichlet and symmetry conditions at the outer boundaries of the domain

$$
\begin{aligned}
& n_{k}^{a}(x=L+q)=N_{k}^{a}, \quad n_{k}^{b}(x=-q)=N_{k}^{b}, k \in\{0,1\}, \\
& \phi^{b}(x=-q)=0, \\
& \partial \phi^{s} / \partial y=\partial n_{k}^{s} / \partial y=0 \text { at } y=0, y=h+H / 2,
\end{aligned}
$$

where $q$ is the thickness of the apical and the basal regions (see Figure 2) and $N_{k}^{a}, N_{k}^{b}, k \in$ $\{0,1,2\}$ are the imposed ion concentrations at the top of the apical and the bottom of the basal regions, respectively. We wish to predict the value of TEP for the comparison of the model results with in-vitro experimental measurements. In particular, TEP is normally determined experimentally with open circuit conditions, i.e. by imposing zero transepithelial current [38]. This can be achieved by imposing a Neumann condition of zero electrical current, which we set at the top of apical region, so that

$$
\frac{\partial \phi^{a}}{\partial x}=0, \quad \text { at } x=L+q
$$

At the boundary between the basal region and the cleft gap we impose continuity of variables and fluxes.

To summarise, in every region $s=a, b, i, g$ there are three equations, $1 \mathrm{a}$ for $\mathrm{Na}^{+}$and $\mathrm{K}^{+}(k=0,1)$ and $(3)$ for the electrical potential. Noting the geometry of Fig. 2 we have 
$k=0,1$ and (5), making 24 conditions in total. We also have 5 Dirichlet 6a -6b), one Neumann (6d) and 18 symmetry conditions $6 \mathrm{bc}$ at the outer boundaries of the domain and 6 conditions at the boundary between the basal region and the cleft gap. Therefore in total we have 54 conditions. However, the lower boundary of the apical region $x=L$ consists of the boundary with the tight junction for $0 \leq y<h$ and with the cell for $h \leq y \leq H / 2+h$, and we can describe the conditions on the corresponding regions as a single $y$-dependent boundary condition at $x=L$. This merging allows us to reduce the number of effective boundary conditions to 51 . The same argument is valid at the basal region for the boundary $x=0$, reducing the number of boundary conditions to 48 . Finally, we have a second order problem in four rectangles, having a single condition at each side of the rectangle, leading to a well defined system of 12 nonlinear coupled second order PDEs for 12 unknowns in the regions with four boundaries, and also 48 boundary conditions. The chloride concentration $n_{2}^{s}$ is found from the electroneutrality condition [2].

\subsubsection{Ion fluxes through cell membranes}

In this section we describe the choice of constitutive relations for the currents across the cell membranes. We consider the components for the ion transport across the RPE as shown in Fig. 2 and discussed in Section 2.1. Ion fluxes through the membranes take into account both active and passive currents. We model the diffusive fluxes through the $k$ ion channel across the membrane from region $s$ to region $m$ with the GoldmanHodgkin-Katz (GHK) flux equation [39]

$$
\mathscr{J}_{k}^{s m}=z_{k} P_{k}^{s} \frac{F}{R T} V^{s m} \frac{n_{k}^{s}-n_{k}^{m} \exp \left(-\frac{z_{k} F V^{s m}}{R T}\right)}{1-\exp \left(-\frac{z_{k} F V^{s m}}{R T}\right)},
$$

where $V^{s m}=\phi^{s}-\phi^{m}$ is the potential across membrane $s$; for the meaning and values of all constants please refer to Table 2 .

To model the $\mathrm{Na}^{+} / \mathrm{K}^{+}$-ATPase and $\mathrm{Na}^{+} / \mathrm{K}^{+} / 2 \mathrm{Cl}^{-}$co-transporter fluxes through the apical membrane we use relations as in the work of Strieter et al [40]. The flux 
from $\mathrm{Na}^{+} / \mathrm{K}^{+}$-ATPase is given by

$$
\mathscr{P}=P\left(\frac{n_{0}^{i}}{K_{N a}+n_{0}^{i}}\right)^{3}\left(\frac{n_{1}^{a}}{K_{K}+n_{1}^{a}}\right)^{2}
$$

where $K_{N a}=0.2\left(1+n_{1}^{i} / 8.33\right) \mathrm{mM}$ and $K_{K}=0.1\left(1+n_{0}^{a} / 18.5\right) \mathrm{mM}$ are apparent $\mathrm{Na}^{+}$ and $\mathrm{K}^{+}$dissociation constants (see [40]) and $P$ is the maximum flux through the pumps.

The model for NKCC co-transporter flux can be linked to free energy as described in [40]. This approach has an advantage with respect to kinetic models, as the only parameter to estimate is the co-transporter coefficient $M$. The flux through NKCC channels has the following expression

$$
\mathscr{M}=M \log \frac{n_{0}^{i} n_{1}^{i}\left(n_{2}^{i}\right)^{2}}{n_{0}^{a} n_{1}^{a}\left(n_{2}^{a}\right)^{2}} .
$$

The fluxes through the membranes for every ion can therefore be rewritten as follows

$$
\begin{aligned}
J_{0}^{i a}=3 \mathscr{P}+\mathscr{M}, & J_{0}^{i b}=0, \\
J_{1}^{i a}=\mathscr{J}_{1}^{i a}-2 \mathscr{P}+\mathscr{M}, & J_{1}^{i b}=\mathscr{J}_{1}^{i b}, \\
J_{2}^{i a}=2 \mathscr{M}, & J_{2}^{i b}=\mathscr{J}_{2}^{i b},
\end{aligned}
$$

where $b$ denotes both basal and lateral membranes. The tight junction is modelled as a membrane which is permeable only to $\mathrm{K}^{+}$and the fluxes are modelled using the GHK model

$$
J_{1}^{a g}=\mathscr{J}_{1}^{a g}
$$

Finally, the current of ion $k$ at the membrane between the regions $s$ and $m$ is given by the expression $I_{k}^{s m}=z_{k} F J_{k}^{s m}$.

\subsubsection{Model reduction}

To simplify the system we take advantage of the fact that the cleft gap has a very small aspect ratio $\delta=h / L \approx 0.001$. This allows us to make some assumptions that are all verified a posteriori with a direct numerical solution in Comsol Multiphysics [41]. 
The first simplification of the system (1a), (3)- $6 \mathrm{cc}$ ) is to neglect all the spatial variations in the apical and basal regions. This is not obviously true in the vicinity of the tight junction in the apical region and the boundary with the cleft gap in the basal one. However, the numerical simulations in Comsol Multiphysics show that the relative deviations of concentrations and the potential in the apical and basal regions from the imposed values at $x=-q$ and $x=L+q$ are of the order of $1-2 \%$. We, therefore, consider all the model variables in the apical and basal region to be uniform and equal to the values at the boundaries $(6 \mathrm{a}),(6 \mathrm{~b})$ and impose the zero current condition at the apical membrane, so that it reads

$$
\mathscr{P}+\mathscr{J}_{1}^{i a}+2 \delta \mathscr{J}_{1}^{g a}=0 .
$$

Since water transport across the RPE is isotonic [10] we assume that there is no concentration jump across the epithelium, and thus osmotic flux can occur only due to local osmosis through the cleft gap, implying that the lateral fluxes of water and ions have the same order of magnitude as the fluxes through the cell. This in turn means that the flux per unit area emerging from the cleft is $1 / \delta$ times larger than the one supported by the cell. This implies that the spatial variability in the cell is negligible compared to the cleft (diffusion being strong enough to suppress ionic concentration gradients) and suggests that all intracellular variables can be considered as uniform and can be found by solving a spatially averaged problem. In fact, a posteriori estimates show that the potential and concentration changes in the cell have an order of magnitude of less than $1 \%$ of the corresponding average values. Following the above reasoning, the flux balance Eq. (1a) for $\mathrm{Na}^{+}$and $\mathrm{K}^{+}$and the equation for electrical potential (3) in the cell are simplified to a set of algebraic equations for the averaged quantities (see Appendix A for the derivation)

$$
\begin{aligned}
& 3 \mathscr{P}+\mathscr{M}=0, \\
& \mathscr{J}_{1}^{i a}-2 \mathscr{P}+\mathscr{M}+\mathscr{J}_{1}^{i b}+\frac{2}{H} \int_{0}^{L} \mathscr{J}_{1}^{i g} d x=0, \\
& \mathscr{P}+\mathscr{J}_{1}^{i a}+\mathscr{J}_{1}^{i b}-\mathscr{J}_{2}^{i b}+\frac{2}{H} \int_{0}^{L}\left(\mathscr{J}_{1}^{i g}-\mathscr{J}_{2}^{i g}\right) d x=0 .
\end{aligned}
$$


For the meaning of all other symbols and variables refer to Table 1 and 2 .

Taking advantage of the fact that the cleft is long and thin, the equations there can also be simplified by using an asymptotic expansion in terms of $\delta$, which leads to a set of three coupled ODEs (derived in Appendix B

$$
\begin{aligned}
& D\left(\frac{d^{2} n_{0}^{g}}{d x^{2}}+\frac{d}{d x}\left(\frac{F}{R T} n_{0}^{g} \frac{d \phi^{g}}{d x}\right)\right)-\left(q \frac{d n_{0}^{g}}{d x}-Q^{g} n_{0}^{g}\right)=0, \\
& D\left(\frac{d^{2} n_{1}^{g}}{d x^{2}}+\frac{d}{d x}\left(\frac{F}{R T} n_{1}^{g} \frac{d \phi^{g}}{d x}\right)\right)-\left(q \frac{d n_{1}^{g}}{d x}-Q^{g} n_{1}^{g}\right)+\mathscr{J}_{1}^{i g}=0, \\
& 2 \frac{D F}{R T} \frac{d}{d x}\left(\left(n_{0}^{g}+n_{1}^{g}\right) \frac{d \phi^{g}}{d x}\right)+\mathscr{J}_{1}^{i g}-\mathscr{J}_{2}^{i g}=0,
\end{aligned}
$$

where $q=\frac{1}{2 h} \int_{0}^{2 h} u d y$ is depth-averaged velocity and $Q^{g}$ is the water flux per unit surface area through the lateral membrane, defined in terms of osmotically driven and pressure driven fluxes, as specified in Section 2.3 . The equations governing the velocity field $\mathbf{u}=(u, v)$ are also described in Section 2.3

For boundary conditions at the tight junction $(x=L)$ we impose the influx of $\mathrm{K}^{+}$ with zero-flux for other ions

$$
\begin{aligned}
& D\left(\frac{d n_{0}^{g}}{d x}+\frac{F}{R T} n_{0}^{g} \frac{d \phi^{g}}{d x}\right)-Q^{t j} n_{0}^{g}=0, \\
& D\left(\frac{d n_{1}^{g}}{d x}+\frac{F}{R T} n_{1}^{g} \frac{d \phi^{g}}{d x}\right)-Q^{t j} n_{1}^{g}=\mathscr{J}_{1}^{a g}, \\
& \frac{2 D F}{R T}\left(n_{0}^{g}+n_{1}^{g}\right) \frac{d \phi^{g}}{d x}=\mathscr{J}_{1}^{a g} \quad \text { at } x=L,
\end{aligned}
$$

where $Q^{t j}$ is the water flux per unit surface through the tight junction. At the border with the basal region $(x=0)$ we prescribe Dirichlet conditions, $n_{k}^{g}(x=0)=N_{k}^{b}, k=0,1$ and $\phi^{g}(x=0)=0$. The $\mathrm{Cl}^{-}$concentration can be found from the electroneutrality condition (2). For the definition and meaning of all the symbols please refer to Table 2 .

We therefore have reduced the nonlinear coupled system (1a), (3) - 6d to a system of algebraic equations (12)-(13) for the variables in the cell and TEP, coupled with three ODEs 14 for $\mathrm{Na}^{+}, \mathrm{K}^{+}$concentrations and the potential in the cleft gap. In the above set of equations the concentration of fixed negative charge $X^{i}$ is unknown and is a surrogate for the unknown cell volume; to determine it we need to impose an additional 
equation, which comes from water balance and will be discussed in Section 2.4. This model of ion transport is the basis to model osmotic flow. However, more information is needed to model electroosmosis, as we need to resolve the Debye layers in the cleft gap.

\subsubsection{The electrical double layer}

In the electrical double layer (EDL), electroneutrality is not valid and the full system (1) in the gap $(s=g)$ has to be solved. The boundary conditions (4) for the bulk variables are unchanged. However, we need an additional condition at the membrane for the electrical potential. This condition imposes that the component of the electrical field normal to the membrane equals the surface charge density over the dielectric permittivity

$$
\frac{\partial \phi^{E D L}}{\partial y}=\frac{C_{m}\left(\phi^{g}-\phi^{i}\right)-\sigma_{0}}{\varepsilon} \quad \text { at } y=h, x \in[0, L] .
$$

In the above expression $C_{m}\left(\phi^{g}-\phi^{i}\right)$ is the Debye layer stored charge associated with capacitance and $\sigma_{0}$ is the wall charge. The former is related to the electrical potential difference between the bulk of the cell and cleft via the membrane capacitance per unit area, $C_{m}$, while the latter is the fixed charge due to the presence of charged membrane proteins.

At the boundary of the EDL and the bulk of the cleft we match the solution with the corresponding values in the bulk.

The model can be simplified due to the small aspect ratio between the electrical double layer and the cleft gap length, $\lambda=\lambda_{D} / L \approx 1.8 \cdot 10^{-4}$, using an asymptotic expansion in terms of $\lambda$; the analytical solution for the leading-order problem, in terms of the ion concentrations $n_{k}^{E D L}, k=0,1,2$ and the potential $\phi^{E D L}$ in the EDL is derived in Appendix C. We note that at the leading order the advection is subdominant in the EDL and is thus neglected. This solution allows us to estimate the value of the slip velocity for the electroosmotic flux in the cleft, as discussed in the following section.

\subsection{Flow in the cleft gap}

We finally investigate water flow in the cleft gap driven by electroosmosis, osmotic and mechanical pressure differences. The solution is constructed by using a boundary- 
layer theory, with the inner region corresponding to the EDL (similar to the works of [42, 43]) and the outer one, the bulk of the cleft gap. The analysis requires separation of scales between the thickness of the cleft gap and the EDL, i.e. $\lambda / \delta \ll 1$. This ratio is 0.18 , which, given that the quantities in the EDL are decaying exponentially fast (as in (C.9)), is sufficiently accurate for our objectives. Moreover, the error of this approximation is $(\lambda / \delta)^{2} \approx 0.03$. Matching between the solutions in the two regions provides us with an expression for the slip velocity to be used later as a boundary condition in the outer problem.

In the inner region (the EDL), the presence of non-zero net charge density is accounted for in the context of fluid mechanics as a body force in the inertialess fluid momentum balance. Therefore the problem is governed by the following system

$$
\begin{aligned}
& -\nabla p+\mu \nabla^{2} \mathbf{u}+\varepsilon\left(\nabla^{2} \Phi\right) \nabla \Phi=0 \\
& \nabla \cdot \mathbf{u}=0
\end{aligned}
$$

where $\mathbf{u}=(u, v)$ is fluid velocity, $p$ is pressure and $\Phi$ is the potential in the cleft gap, obtained as a composite solution by matching the potential in the EDL and in the bulk.

The problem in the outer region (the bulk of the cleft gap, away from the cell membrane) is governed by the standard Stokes equations, i.e. 17a, 17b without the body force term. The matching procedure is reported in Appendix D and results in the following expression for the slip velocity at the boundary between the EDL and the bulk of the cleft:

$$
u_{\text {slip }}=-\kappa \varepsilon \frac{C_{m}\left(\phi^{g}-\phi^{i}\right)-\sigma_{0}}{\mu \sqrt{C(x)}} \frac{\partial \phi^{g}}{\partial x}-\kappa^{2} \varepsilon C^{\prime}(x) \frac{\left(C_{m}\left(\phi^{g}-\phi^{i}\right)-\sigma_{0}\right)^{2}}{8 \mu C^{2}(x)}
$$

where $C(x)=\sum_{k=0}^{2} n_{k}^{g}$ and $\kappa=\sqrt{R T / \varepsilon F^{2}}$. The first term of the above expression is analogous to the standard Helmholtz-Smoluchovsky slip velocity [21] for variable electric field and zeta potential; the second term appears due to the fact that the concentrations in the bulk of the cleft are non-uniform.

We use this velocity as the slip boundary condition at the lateral walls to solve the problem in the bulk. We assume that hydrostatic pressure difference across the cell 
layer and across cell membranes (but not tight junctions) is negligible [10]. Thus, without loss of generality, we can set $p$ equal to zero in the cell, apical and basal regions, and hence $p$ measures the pressure relative to its average values in these regions. We consider the presence of osmotic and pressure-driven fluxes through the tight junction $Q^{t j}$ and lateral membranes $Q^{g}$, which are given by the following expressions

$$
\begin{aligned}
& Q^{g}(x)=k_{m}\left[R T\left(\sum_{k=0}^{2}\left(n_{k}^{i}-n_{k}^{g}(x)\right)+X\right)+p(x)\right], \\
& Q^{t j}=k_{t j}\left[R T \sum_{k=0}^{2}\left(n_{k}^{a}-n_{k}^{g}(L)\right)+p(L)\right]
\end{aligned}
$$

where $k_{m}$ and $k_{t j}$ are the hydrodynamic permeabilities of the cell membrane and the tight junction respectively. Note that all reflection coefficients in this and following similar expressions are taken to be unity as is reported to be typical in the context of ion transport for animal cell homoeostasis [34]. Eq. [17] is, therefore, reduced using lubrication theory, to give the water velocity in the bulk as

$$
u=\frac{1}{2 \mu} \frac{\partial p}{\partial x}\left(y^{2}-h^{2}\right)+u_{\text {slip }}, \quad-h \leq y \leq h, \quad 0 \leq x \leq L
$$

(the trans-cleft coordinate $y$ is illustrated in Fig. 2). The equation for the pressure is obtained by integrating the continuity equation, to give

$$
-\frac{h^{3}}{3 \mu} \frac{d^{2} p}{d x^{2}}+h \frac{d u_{s l i p}}{d x}=-Q^{g},
$$

subject to boundary conditions

$$
\begin{aligned}
& p=0 \quad \text { at } x=0, \\
& -\frac{h^{3}}{3 \mu} \frac{d p}{d x}+h u_{\text {slip }}=Q^{t j} \quad \text { at } x=L .
\end{aligned}
$$

Equation 22a balances the gradient of fluid flux along the cleft with osmotic transport driven by pressure differences across the lateral walls. Finally, the $y$-component of the velocity $v$ can be obtained from $(17 b)$. The derivation of the above expressions is 


\subsection{Coupling between ion and fluid flow}

We now have a model for ion and fluid transport. To couple the two we write the flux-balance equation in the cell as

$$
\int_{0}^{H} Q^{a} d y+\int_{0}^{H} Q^{b} d y+2 \int_{0}^{L} Q^{g} d x=0
$$

where the fluid fluxes $Q^{a}, Q^{b}$ are defined as

$$
Q^{a}=A_{f} k_{m} R T\left(\sum_{k=0}^{2}\left(n_{k}^{i}-n_{k}^{a}\right)+X\right), \quad Q^{b}=k_{m} R T\left(\sum_{k=0}^{2}\left(n_{k}^{i}-n_{k}^{b}\right)+X\right) .
$$
transport.

\subsection{Solving the fully coupled problem}

We proceed to summarise how ion and fluid dynamics are coupled in our model and how the solution, including the water balance constraint, is obtained.

335

The first step of the model is to solve the problem for ion transport in the cell numerically (13), imposing an initial value of $X$, to obtain values for $T E P, \phi^{i}, n_{0}^{i}$ and $n_{1}^{i}$. These quantities appear in boundary conditions for the problem in the cleft and, therefore, also in Eqs. (14). Resolving the double layer is essential to obtain the expression for the slip velocity (18), which appears in the problem for the fluid in the cleft 222. The slip velocity depends on the bulk concentrations and the potential in the cleft gap. The problem for ion transport in the cleft (14) is thus coupled with fluid transport 22) via advection, osmotic water fluxes and the slip velocity. They are solved 
numerically together to obtain the profiles of concentrations $n_{k}^{g}, k=0,1$, the potential $\phi^{g}$ in the bulk of the cleft, fluid velocity $\mathbf{u}$ and pressure $p$.

The last step of the solution is to enforce water flux balance in the cell, which is given by equation 23. This condition is satisfied by recalculating the value of $X$ and setting up an iterative procedure with respect to $X$, until water balance is enforced with the required precision. This is implemented by the following steps:

1. For a given value of $X$, denoted $X_{\text {prior }}$, we solve the spatially-averaged problem for ion transport (13) for the unknowns TEP, $\phi^{i}, n_{0}^{i}$ and $n_{1}^{i}$ in the cell.

2. We solve the problem for fluid and ion transport in the bulk of the cleft (14), 22) to find the distributions of potential $\phi^{g}$, concentrations $n_{0}^{g}$ and $n_{1}^{g}$, fluid pressure $p$ and velocity $\mathbf{u}$, using the expression for the slip velocity [18].

3. We find the fluxes $Q^{s}, s \in\{a, b, g, t j\}$ and use (23) to solve for the iteration update of $X$, that is $X_{\text {update }}$.

4. We go to step 1 with the updated value of $X$, now resetting $X_{\text {prior }}=X_{\text {update }}$, for the next iteration until the condition (23) is satisfied with $X_{\text {prior }}$ for a desired tolerance.

This solution procedure was implemented in Matlab to obtain all the results reported in Section 4

\section{Choice of the parameter values}

The choice of parameters is very challenging due to the lack of clinical measurements on human RPE. Some data on ion fluxes and membrane permeabilities have been reported for other species, such as frog and cow, but to our knowledge, no such measurements are available for humans. We thus were forced to make assumptions on some of the parameter values. As a general rule, we tried to use data from the literature whenever possible and attempted to reproduce with the model concentrations of the various species and TEP close to measured values. Since the model is a simplification of the real system and not all ingredients are considered, the values of some parameters we use might need adjustment in more refined models. The list of reference parameters used in the model is reported in Table 2 and their choice is discussed below. 


\subsection{Geometry}

We consider the RPE as a monolayer of rectangular cells with thickness $H$ and length $L$. The typical RPE length varies from 10 to $60 \mu \mathrm{m}$ [27], with the lower value at

$L=H=10 \mu \mathrm{m}$. The reference width of the cleft gap $h$ is fixed to be $20 \mathrm{~nm}$, noting that the resulting prediction for the net water flux with the other reference parameters matches observed values of $\approx 10^{-8} \mathrm{~m} / \mathrm{s}[31]$. The apical membrane of the RPE cell has long microvilli [2], and the folding factor $A_{f}$ was estimated by Adijanto et al. [23] in

\subsection{Ion transport}

We consider the RPE to be in a bath of $120 \mathrm{mM} \mathrm{NaCl}$ and $5 \mathrm{mM} \mathrm{KCl}$ solution, giving a total osmolarity of $250 \mathrm{mM}$. Therefore, ion concentrations in the apical and basal regions are $N_{0}^{s}=120 \mathrm{mM}, N_{1}^{s}=5 \mathrm{mM}$ and $N_{2}^{s}=125 \mathrm{mM}, s \in\{a, b\}$. In our model we neglect the presence of $\mathrm{HCO}_{3}^{-}$and all the active and passive transport associated with it, such as the $\mathrm{Na}^{+}-\mathrm{HCO}_{3}^{-}$co-transporter and $\mathrm{HCO}_{3}^{-}-\mathrm{Cl}^{-}$exchanger in the basolateral one. Since we do not consider passive transport of $\mathrm{Na}^{+}$across membranes and $\mathrm{Cl}^{-}$across the apical membrane, the corresponding permeabilities are set to be zero, $P_{0}^{a}=P_{0}^{b}=P_{2}^{a}=0$.

$\mathrm{K}^{+}$channels in the apical membrane were studied by Kusaka et al. [44] in the rat RPE, who found the ratio between conductance and cell capacitance to be $g_{1}^{a} / C_{m} \approx$ $0.14 \mathrm{nS} / \mathrm{pF}$, from which we can compute, using the value of $C_{m}$ reported in Table $2, g_{1}^{a} \approx$ 1.4 S $/ \mathrm{m}^{2}$. Using linear expression for the current, one would write $I_{1}^{a i}=g_{1}^{a}\left(\phi^{a}-\phi^{i}+R T / F \ln \left(n_{1}^{a} / n_{1}^{i}\right)\right)$. However, we use the GHK model for the current (7) and estimate the order of magnitude of membrane permeability $P_{1}^{a}$, we match the two expressions for the following values of potential and concentrations: $n_{1}^{i}=120 \mathrm{mM}, \phi^{i}=-75 \mathrm{mV}$ and $\phi^{a}=15 \mathrm{mV}$, and obtain $2.5 \cdot 10^{-8} \mathrm{~m} / \mathrm{s}$. To account for apical membrane folding this value has to be multiplied by 10 . In the following we use $P_{1}^{a} \approx 5 \cdot 10^{-7} \mathrm{~m} / \mathrm{s}$. In our framework, increasing this permeability does not cause significant change of ion concentrations in 
The basolateral membrane permeability to $\mathrm{Cl}^{-}, P_{2}^{b}$, is controlled by the presence of $\mathrm{Cl}^{-}$channels [6]. The conductance of this membrane was estimated in the frog RPE by La Cour [45] to be $\approx 5.9 \mathrm{~S} / \mathrm{m}^{2}$. The value refers to the whole epithelium rather than the membrane itself, and thus the presence of lateral membranes is incorporated into this value, which leads to an estimate of the permeability $\approx 2.7 \cdot 10^{-8} \mathrm{~m} / \mathrm{s}$ via similar calculation as for $P_{1}^{a}$ with $n_{2}^{i}=50 \mathrm{mM}$. Increasing $P_{2}^{b}$ results in depolarisation of basolateral membrane, elevation of $n_{0}^{i}$, decrease of $n_{1}^{i}$ and $n_{2}^{i}$ and the increase of water flux. To ensure the TEP is within the range of observed values [6] we have fixed $P_{2}^{b}=0.8 \cdot 10^{-8} \mathrm{~m} / \mathrm{s}$. For same reasons, the basolateral permeability to $\mathrm{K}^{+}, P_{1}^{b}$, was fixed to be $P_{1}^{b}=1.5 \cdot 10^{-7} \mathrm{~m} / \mathrm{s}$. Increasing permeability $P_{1}^{b}$ results in polarisation of the basolateral membrane and decrease f TEP.

We consider tight junctions to be permeable to $\mathrm{K}^{+}$, based on the observations of Peng et al. [26]. The value of this permeability $P^{t j}$ is unknown, we therefore investigate the effect of variation of this parameter in the Section 4 (see Figs. 4, 6.a)) and take the reference value for the permeability to be $P^{t j}=10^{-6} \mathrm{~m} / \mathrm{s}$.

The order of magnitude for the amplitude of the $\mathrm{Na}^{+}-\mathrm{K}^{+}$pump, $P$, has been chosen based on the work of McLaughlin \& Mathias [30], where they estimated the current from $\mathrm{Na}^{+}-\mathrm{K}^{+}$ATPase to be $10 \mu \mathrm{A} / \mathrm{cm}^{2}$. We fix the maximum value of the pump to correspond to this current, $P \approx 10^{-6} \mathrm{~mol} / \mathrm{m}^{2} / \mathrm{s}$. Increasing the pump rate results in the elevation of TEP and the increase of water flux across the RPE.

In equation (9), the co-transporter coefficient, $M$, is assumed to be triple that of the pump, $M=P \cdot 3 \approx 3 \cdot 10^{-6} \mathrm{~mol} / \mathrm{m}^{2} / \mathrm{s}$ to obtain a $\mathrm{Cl}^{-}$concentration in cell in the range of observed values in frog and bovine RPE [46, 47]. Increasing $M$ results in depolarisation of the basolateral membrane.

The average valence of negatively charged large molecules in the cell, $z_{X}$, differs among cells and species. Lew et al. [48] calculated it to be 1.85 with parameters from amphibian skin and urinary bladder. In this work we fix $z_{X}=1.5$.

Finally, for the solution in the EDL we need to fix the surface charge due to proteins, $\sigma_{0}$. We fix it to correspond to a zeta potential of $-10 \mathrm{mV}$, which is in the range of standard values for the cell membrane [49]. 


\subsection{Fluid dynamics}

We consider intracellular and extracellular fluid to have the properties of water. The electric permittivity $\varepsilon$ is used for $250 \mathrm{mM} \mathrm{NaCl}$ solution at $35^{\circ} \mathrm{C}$ [28]. Water is transported across the cell membrane through a lipid bilayer, which has low permeability, and water channels, aquaporins AQP1 [50] (and other AQPs [51]), that elevate the hydrodynamic permeability. In fact, for some epithelia, the water velocity scale across the membrane can be elevated from $10^{-4} \mathrm{~cm} / \mathrm{s}$ to $10^{-2} \mathrm{~cm} / \mathrm{s}$ by aquaporins [14]. In our model we consider the reference value for membrane permeability to be $k_{m}=1.28 \cdot 10^{-12} \mathrm{~m} / \mathrm{s} / \mathrm{Pa}$ [33] and study the dependence of fluxes on its variation in $440 \quad$ Fig. 7 .

The hydrodynamic permeability of the tight junction is chosen to be 100 times that of the reference value for the membrane permeability, $k_{m}$, so that $k_{t j}=1.28$. $10^{-10} \mathrm{~m} / \mathrm{s} / \mathrm{Pa}$. The variation of this parameter is also shown in Fig. 7

\section{Results}

Throughout this section we use, as baseline, the parameter values reported in Table 2, unless otherwise specified. As discussed in section 2, the concentration of all species in the cell are assumed to be spatially uniform. The values predicted by the ion transport model (13) under open circuit condition 12 are shown in Fig. 2 (left panel) in purple. The values of the concentrations in the apical and the basal regions $N_{k}^{s}, k=0,1,2, s \in\{a, b\}$ are prescribed and are reported in the figure in black. The concentrations predicted for $\mathrm{Na}^{+}, \mathrm{K}^{+}$and $\mathrm{Cl}^{-}$are $n_{0}^{i}=18.5 \mathrm{mM}, n_{1}^{i}=121.5 \mathrm{mM}$ and $n_{2}^{i}=51 \mathrm{mM}$, respectively. All calculated values are close to those observed in the RPE $[23,6,8]$. The existing measurements of $\mathrm{Cl}^{-}$concentration provide values varying in a relatively wide range, from $62 \mathrm{mM}$ in the bovine RPE [47] to approximately $20 \mathrm{mM}$ in the toad and chick RPE [46, 52]. We note that the $\mathrm{Cl}^{-}$values reported are high compared to the other mammalian cell types.

In Fig. 2 we also report the predicted values of the potential in all regions. The potential is, without loss of generality, imposed in the basal region (reported in black) and computed in the other two (interior of the cell and the apical region). The transep- 


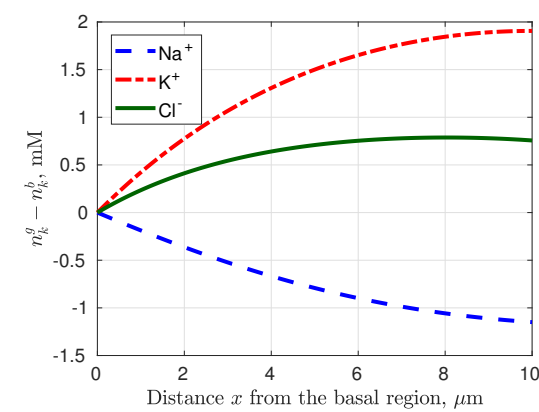

(a)

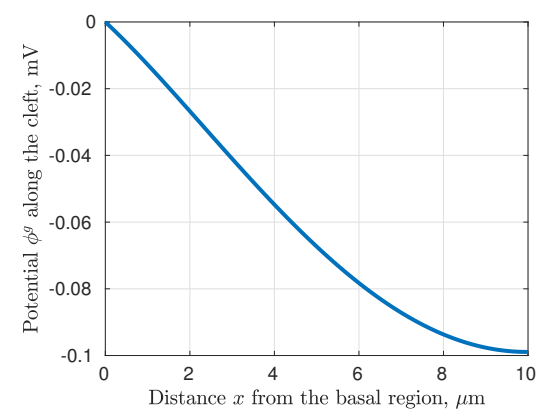

(b)

Figure 3: (a) Deviation of the ion concentrations along the cleft from those in the basal region. The $x$ - axis goes along the symmetry midline $(y=0)$ of the cleft from the basal region to the tight junction, as shown in Figure 2 (b) The electrical potential along the cleft. $P^{t j}=10^{-6} \mathrm{~m} / \mathrm{s}, \delta=10^{-3}$. All other parameters are given in Table 2

ithelial potential TEP $=\phi^{a}-\phi^{b}$ is positive and equal to $\approx 14.6 \mathrm{mV}$, which is within the range of values observed in different species, from $2.2 \mathrm{mV}$ to $15 \mathrm{mV}$ [6, 53, 54].

Under open circuit condition, $\mathrm{K}^{+}$and $\mathrm{Cl}^{-}$fluxes are about $2.96 \cdot 10^{-6} \mathrm{~mol} / \mathrm{m}^{2} / \mathrm{s}$. There is no net $\mathrm{Na}^{+}$transport, since we do not consider the $\mathrm{Na}^{+}-\mathrm{HCO}_{3}{ }^{-}$co-transporter in the basolateral membrane [10]. The value of $\mathrm{Cl}^{-}$flux predicted by our model is larger than the one measured by La Cour [45] in the frog RPE, which was $\approx 2$. $10^{-6} \mathrm{~mol} / \mathrm{m}^{2} / \mathrm{s}$, but has the same order of magnitude.

Ion fluxes occur from the cell both to the basal region and the cleft gap. The flux through the lateral wall has to be balanced by a flux along the cleft, which is supported by ion concentration and electrical potential gradients. In Fig. 3 a) we plot the departure of the concentration of all species from their basal values along the cleft. $\mathrm{K}^{+}$ and $\mathrm{Cl}^{-}$concentrations increase towards the tight junction. The $\mathrm{Na}^{+}$concentration, on the other hand, is decreasing and, since lateral membranes are impermeable to it, the behaviour is governed by electroneutrality. The variation of concentration has the same order of magnitude for all species. The potential along the cleft gap is shown in Fig. 3 (b). The figure shows that the potential decreases from zero at the basal region to a negative value at the tight junction, despite the fact that the apical potential is positive. This implies that the potential drop across the tight junction has a larger magnitude than the TEP. 


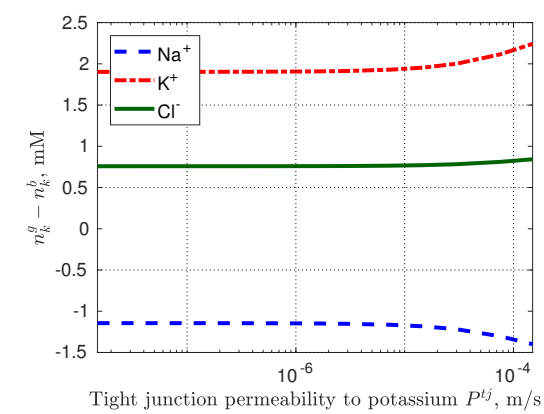

(a)

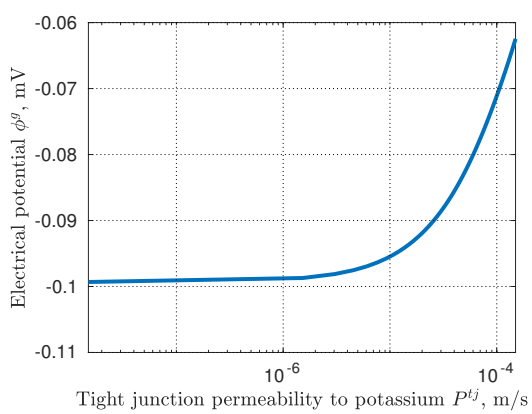

(b)

Figure 4: Differences in ion concentrations (a) and the potential (b) between model predictions in the cleft at the boundary with the tight junction and at the basal region, as a function of tight junction permeability to $\mathrm{K}^{+}, P^{t j}$. All other parameters are given in Table 2

The value of tight-junction permeability to $\mathrm{K}^{+}, P^{t j}$, appearing in the Eq. [11], is un-

480

known in the RPE, and in other epithelia it could vary over several orders of magnitude, depending whether the epithelium is leaky or tight [55]. The drop of ion concentrations and of the potential in the cleft is shown in Fig. 4. We vary $P^{t j}$ from $1.5 \cdot 10^{-8}$ to $1.5 \cdot 10^{-4} \mathrm{~m} / \mathrm{s}$. Within this range (4 orders of magnitude) the concentrations and the potential remain almost constant, implying our lack of knowledge about the permeability of the tight junction does not significantly affect the model results.

The concentration variation of all species in the cleft, along with the fact that the concentrations in the cell are not spatially variable, implies that an osmotic flux is generated across the cell lateral membrane. Such a flux is directed from the cell to the cleft, since the osmolarity in the cleft is larger than in the cell. Moreover, the existence of an electrical field along the cleft generates an electroosmotic flow. Since electroosmotic flow follows the potential gradient, it is directed from the basal to the apical region and therefore competes with the osmotic flux.

The velocity field in the cleft is shown in Fig. 5. a). The effect of the slip velocity can be seen through the circulation next to the walls, close to the boundaries of the cleft with the basal region. The slip velocity is directed from the basal to the apical region, which is a consequence of the negative sign of the potential gradient along the cleft. However, water flow is mostly due to the lateral osmotic fluxes. The inflow from the 


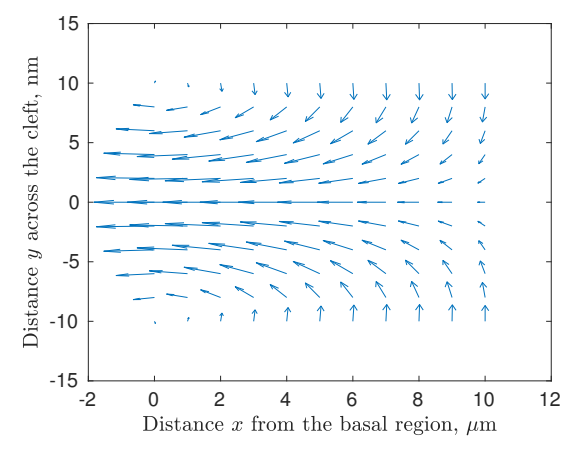

(a)

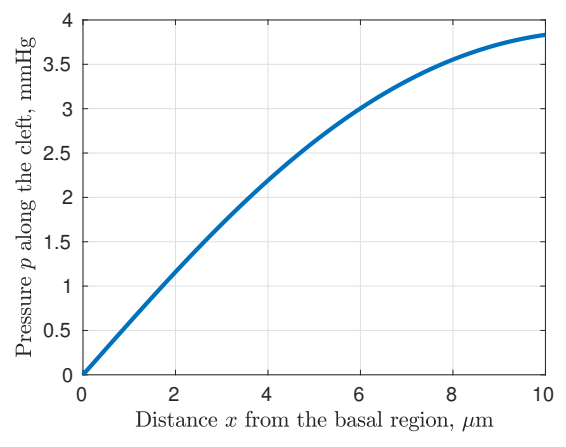

(b)

Figure 5: (a) The velocity vectors in the cleft. The $x$-axis spans from basal to apical region, the $y$-axis spans across the cleft. The cleft thickness is magnified for clearer visualisation. The maximum velocity magnitude is $5.2 \mu \mathrm{m} / \mathrm{s}$. (b) Fluid pressure along the cleft. Parameters are reported in Table 2 with $P^{t j}=10^{-6} \mathrm{~m} / \mathrm{s}$, $\delta=10^{-3}, k_{t j}=1.28 \cdot 10^{-10} \mathrm{~m} / \mathrm{s} / \mathrm{Pa}$.

tight junction is barely visible in the plot because its magnitude is much smaller than the one from the lateral walls.

Figure 5(b) shows the pressure along the cleft gap relative to the pressure in the cell, which we assume to be constant and equal to zero. The maximum value is close to the tight junction and is about $4 \mathrm{mmHg}$. We note that in reality the cleft gap is compliant and such pressure values might induce an increase of its thickness. Introducing this effect to the model would add extra complexity and additional unknown parameters (see [11]), without qualitatively changing the results. For instance, for a cleft gap 5 times thicker, $h=50 \mathrm{~nm}$, the maximum pressure value drops to $0.025 \mathrm{mmHg}$, but the water flux reduces only by a factor of 1.5 . Therefore, similarly to what has been implemented in various other works on epithelial transport (e.g. [56]), we do not account for the compliance in the present model.

The contribution of the electroosmotic flux in the cleft $F_{E O}$ to the total flow is computed via $F_{E O}=F_{T O T}-F_{O}$, where $F_{T O T}$ is the transepithelial flux per unit surface and $F_{O}$ is the flux, driven only by osmosis, obtained by solving [22], with zero slip velocity $u_{\text {slip }}=0$ (thus 'switching off' electroosmosis). The model predicts, that $F_{E O} \approx 0.9 \cdot 10^{-11} \mathrm{~m} / \mathrm{s}$ and $F_{O} \approx 0.7 \cdot 10^{-8} \mathrm{~m} / \mathrm{s}$, indicating that osmosis dominates electroosmosis. The overall flux is therefore directed towards the basal region. In Fig. 2 


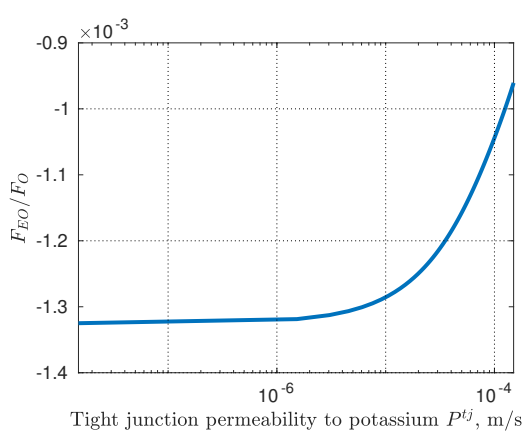

(a)

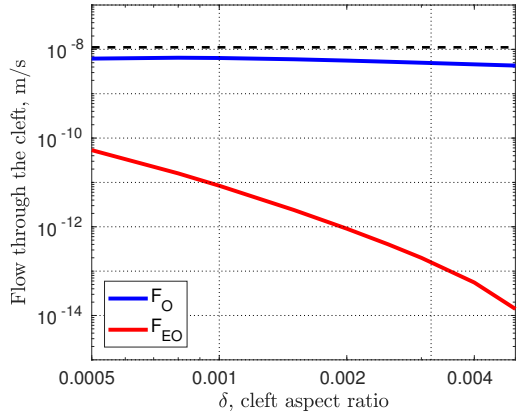

(b)

Figure 6: (a) Ratio between predicted electroosmotic and osmotic fluxes across the RPE as a function of tight junction permeability $P^{t j}$ with $\delta=10^{-3}$. (b) Osmotic flux $F_{O}$ and electroosmotic flux $F_{E O}$ magnitudes as a function of aspect ratio of the cleft $\delta=h / L$ with $P^{t j}=10^{-6} \mathrm{~m} / \mathrm{s}$. The black dashed line corresponds to the measured value of transepithelial water transport $4 \mu \mathrm{l} / \mathrm{h} / \mathrm{cm}^{2} \approx 1.1 \cdot 10^{-8} \mathrm{~m} / \mathrm{s}$ [32]. In both figures $k_{t j}=1.28 \cdot 10^{-10} \mathrm{~m} / \mathrm{s} / \mathrm{Pa}$. All other parameters are given in Table 2

we show the direction of water fluxes predicted by the model. The length of the arrows schematically indicates the magnitude of the corresponding flux. We note that there is an osmotic flux also from the basal region to the cell, but it is smaller than that from the apical region, owing to the fact that the apical membrane is highly folded. For baseline values for the parameters, the water flux per unit surface through the RPE is equal to $0.7 \cdot 10^{-8} \mathrm{~m} / \mathrm{s}$.

The ratio between osmotic and electroosmotic fluxes as a function of the tightjunction permeability to $\mathrm{K}^{+}$is shown in Fig. 6(a). The flux ratio is negative since the electroosmotic flow has an opposite direction to the osmotic flow. Moreover, this ratio keeps the same order of magnitude over a wide range of values of $P^{t j}$, which indicates that electroosmotic flux is subdominant. Fig. 6(b) shows the effect of the cleft aspect ratio $(h / L)$ on the osmotic and electroosmotic contributions to the total flow. Increasing the cleft aspect ratio results in smaller potential and concentration gradients and both fluxes decrease. Electroosmosis becomes more relevant as the aspect ratio tends to zero.

In Fig. 7 we investigate the effect of the hydrodynamic permeability of the lateral membrane $k_{m}$ and of the tight junction $k_{t j}$ on the flow (see Eq. 22a). The five curves correspond to different values of $k_{t j}$ for which, to our knowledge, there are no mea- 


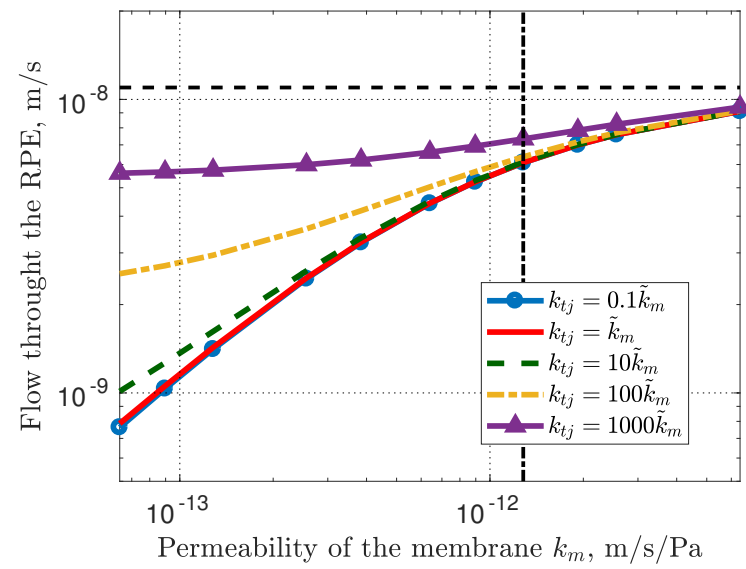

Figure 7: Flux per unit surface through the RPE as a function of the membrane hydrodynamic permeability $k_{m}$ for different values of tight junction hydrodynamic permeability $k_{t j} . \tilde{k}_{m}$ is the typical value for hydrodynamic permeability of the cell membrane, $\tilde{k}_{m}=1.28 \cdot 10^{-12} \mathrm{~m} / \mathrm{s} / \mathrm{Pa}[33]$. The baseline value for the membrane permeability is represented by the vertical black punctured line. The horizontal black dashed line corresponds to the measured value of transepithelial water transport $1.1 \cdot 10^{-8} \mathrm{~m} / \mathrm{s} . \delta=10^{-3}, P^{t j}=10^{-6} \mathrm{~m} / \mathrm{s}$. All other parameters are given in Table 2

surements. We thus consider it to be a multiple of the typical membrane permeability, which we denote as $\tilde{k}_{m}$. Fig. 7 shows that the hydrodynamic permeability of the cell membrane $k_{m}$ has a significant influence on the magnitude of the flow in the cleft, since it governs the osmotic fluxes. On the other hand, due to the small cleft aspect ratio $\delta$, the permeability of the tight junction, $k_{t j}$, has a smaller effect on water flux. $k_{t j}$ should be of order $k_{m} / \delta$ for the osmotic fluxes through the tight junction and lateral walls to have the same magnitude. In fact, it can be seen from the plot that for large values of $k_{t j}$ the flux is weakly affected by changes in $k_{m}$.

\section{Discussion}

A significant proportion of ocular pathology is due to fluid accumulation in the subretinal space. It occurs in diabetic maculopathy and some forms of age-related macular degeneration, the two being among the most common causes of visual impairment or blindness. One of the reasons of fluid accumulation in the sub-retinal space is failure of fluid pumping by the retinal pigment epithelium (RPE). Failure of such a pumping is also associated with increased risk of retinal detachment [4]. Understanding the 
mechanisms of fluid transport across the RPE is important to obtain an insight about preventing and/or treating the pump failure and it is the primary aim of the present work.

The mechanisms that drive fluid across various types of epithelia have been thoroughly investigated [13]. In addition, many authors studied water transport across the corneal endothelium, in particular focusing on the role of electroosmosis [58, 59,

${ }_{555}$ 19]. However, the details of RPE water transport are poorly understood, despite their clinical relevance, though the close integration with ion transport suggests there are favourable prospects for furthering a mechanistic description for water transport in the RPE.

In this work we propose a mathematical model that incorporates this coupling between cellular ion and fluid transport within a coherent framework that resolves ion concentrations and the electric potential within the cleft gap. The model accounts for the presence of three ions $\left(\mathrm{Na}^{+}, \mathrm{K}^{+}\right.$and $\left.\mathrm{Cl}^{-}\right)$, which, according to experimental measurements, are dominant in terms of concentrations and magnitude of ion fluxes across the RPE [24]. Although it neglects some potentially important elements, such as bicarbonate, protons, carbon dioxide and lactate, the three-ion model is anticipated to be sufficient, as a first approximation, to describe the essential features of RPE water and ion transport, as well as illustrating that physically consistent, spatially resolved models for water and ion transport can be developed, which is a further aim of this work. Adding more components to the model will be the next step of our research.

The model of ion transport consists of conservation equations for ion fluxes and the Poisson equation for the electrical potential together with prescribed boundary and membrane conditions. We consider the apical membrane to be flat, disregarding the presence of microvilli in the geometry, but accounting for them in terms of membrane transport properties. In the bulk of the cell and extracellular space we assume electroneutrality. The small aspect ratio of the cleft ( $\delta=h / L \approx 0.001$ ), allows us to significantly simplify the system. As a result, we obtain distributions of concentrations and the potential in the bulk of both the cell and the cleft gap. We also resolve the Debye layers, at the cell membranes, to obtain the spatial profile of potential and charge density in the cleft, which are necessary to describe electroosmotic flow. 
To model the flow in the cleft gap between adjacent cells we account for the effects of osmotic fluxes through the lateral walls and the tight junction and electroosmosis along the cleft gap. The ion transport model provides the necessary information to describe these mechanisms. Taking advantage of the cleft geometry, we use a thin-film approximation to model fluid flow and note that water and ion transport are coupled through the effect of advection. An additional coupling arises from the overall water balance which controls cell volume.

For the chosen set of parameters (given in Table 2), the model reproduces ion concentrations in the cell and membrane potentials close to those observed in the RPE experimentally. The model resolves the heterogeneous profiles in the cleft gap in concert with a coupling of the ion and fluid transport within a coherent framework. Unlike most works on epithelial transport (e.g. [60], [40],[48]), the present model treats the system as fully spatial until and unless there is evidence to neglect spatial variability, and it is found that some spatial effects need to be retained. In particular, the predicted spatial variability of ion concentrations and electrical potential along the cleft gap has important consequences and cannot be predicted by standard compartmental models of epithelial transport.

The importance of spatial variability was already highlighted by Weinstein and Stephenson [11], who developed a mathematical model for general epithelia, with particular attention to Necturus gallbladder. Despite the similarities, our model holds several substantial differences with the work in [11]. First, the authors aimed at investigating the transport in leaky epithelia, whereas the RPE is tight. Second, as a result of distinct cell types in two models, different ion channel composition and, therefore, constitutive laws were adapted. Moreover, our model investigates the possibility of electroosmosis along the cleft, and therefore the electrical double layers close to the cell membranes are accounted for, which was not implemented in [11]. However, the authors also considered the effect of compliance of the cell membranes, that we neglect even if we account for the changes in cell volume, advocating that this additional effect would not change the qualitative and quantitative in terms of water flux conclusions of the present work.

The results show that the transepithelial flow has a direction from the apical to the 
basal region and its magnitude is about $0.7 \cdot 10^{-8} \mathrm{~m} / \mathrm{s}$, which is close to the measured value of the flow through the human RPE of $1.1-2.8 \cdot 10^{-8} \mathrm{~m} / \mathrm{s}$ [32]. If we account for a hexagonal arrangement of the cells, and assuming that $L$ is the side of cell, then the calculated flux value should be multiplied by a factor of $\approx 2 / \sqrt{3}$. Although we of-magnitude calculation, using the Helmholtz-Smoluchovsky streamwise slip velocity expression [21], $u_{\text {slip }}=-\frac{\varepsilon \zeta E}{\mu}$, where $\varepsilon, \zeta, \mu$ are electric permittivity of the fluid, wall zeta potential and fluid viscosity, respectively, and $E$ is the streamwise electric field. The natural estimate for the electric field would be based on the transepithelial potential 640 $T E P \approx 5 \mathrm{mV}$, thus, $E=-T E P / L \approx-500 \mathrm{~V} / \mathrm{m}$ (where $L$ is the length of the cell) with the field directed from the sub-retinal space to the choroid. In reality, it is likely that 
the majority of the potential drop occurs across the tight junction rather then the whole epithelial layer, entailing that the electric field above is overestimated. It is, however, not obvious how to estimate the value of the potential drop along the cleft, thus we use TEP for this order-of-magnitude argument. Using the values of $\varepsilon$ and $\mu$ from Table 2 and the standard value for the zeta potential for the cell walls $\zeta=-10 \mathrm{mV}$ [49], we estimate $u_{\text {slip }} \approx 4.3 \mu \mathrm{m} / \mathrm{s}$, directed towards the choroid and the electroosmotic flux per unit surface $\approx 8.6 \cdot 10^{-9} \mathrm{~m} / \mathrm{s}$, which is comparable to the measured transepithelial flow. This intuitive calculation, however, is misleading and in disagreement with the model outcomes, since for physiological values of the parameters the electric field is predicted to be directed from the basal region to the tight junction, forcing electroosmotic flow towards the subretinal space, in the opposite direction to the water flux across the RPE.

The magnitude of electroosmosis in the cleft is much smaller and it is thus predicted to be subdominant, stressing the importance of the integrated mathematical model for the epithelial transport. Due to the small aspect ratio in the cleft, the impact of the slip velocity on the total flow is much smaller compared to osmotic fluxes through the lateral walls. In fact, the osmotic flux is about three orders of magnitude larger than the electroosmotic one, and this difference is robust with respect to changes of parameter values. We advocate that above conclusions would apply to other tight epithelia, in particular that electroosmosis is subdominant, though the direction of electroosmotic flow might change depending on the characteristics of the epithelium. Clearly, in the case of non-isotonic transport osmosis will be even more dominant. The present model neglects the possible electroosmotic flow in the tight junctions, noting that such mechanisms have been proposed only for leaky epithelia, whilst the predominance of Claudin-3 and Claudin-19 in human RPE suggest tight epithelia, without relatively low permeability tight junctions [62]. We also have neglected the possible effect of Bruch's membrane, which would result in an additional resistance to the flow.

For physiological values of the membrane permeability $\left(\approx 10^{-12} \mathrm{~m} / \mathrm{s} / \mathrm{Pa}[33]\right)$, the majority of the flow occurs due to the osmotic fluxes through the lateral membrane, via the paracellular route. If, however, the lateral hydrodynamic permeability is low, which can occur due to a lack (or knockdown) of aquaporins, the permeability of the tight junction has to be much larger than the membrane permeability in order to support 
the necessary amount of flow, which will then have a paracellular route. Nevertheless, for low or moderate tight junction hydrodynamic permeability, the transepithelial water flux is approximately proportional to the lateral membrane permeability, which means that this model framework cannot immediately be deployed to explain the effect of aquaporin knockdown that occurs in some leaky epithelia [18].

To summarise, our model predicts that the spatial gradient of ion concentrations and potential in the cleft is capable of driving physiological amounts of fluid through the RPE without additional mechanisms. We must emphasise, however, that since the model neglects $\mathrm{pH}$ balance and the presence of $\mathrm{HCO}_{3}{ }^{-}$and $\mathrm{CO}_{2}$, some of the conclusions might change when adding these components, which is currently the subject of our research. Nonetheless, we anticipate that the presence of spatial heterogeneity in the cleft, and its influence on driving flow across the lateral membranes, will be retained, as will the prediction that electroosmosis is subdominant and the prediction that the water transport may not be as sensitive to membrane permeability as one might initially anticipate.

\section{Appendix A. Reduction of the model in the cell to a set of algebraic equations}

In this section we describe the procedure to simplify the model $1 \mathrm{a}$ for $k=0,1$ and (3) in the cell to a set of algebraic equations. The aspect ratio in the cleft is $\delta=h / L \approx$ 0.001. If we denote schematically the order of magnitude of an ion flux per unit area through the lateral membrane by $\mathscr{F}$, the flux per unit area along the cleft has to be of the order $\mathscr{F} / \delta$. This implies that variations of concentrations and potential in the cell have to be $\delta$ times smaller than those in the cleft. We also verify a posteriori that the magnitude of these variations is of order $0.1 \%$ of the average values. We thus make use of an asymptotic expansion of all variables in the cell and write

$$
n_{k}^{i}=\hat{n}_{k}^{i}+\mathscr{O}(\delta), \quad \phi^{i}=\hat{\phi}^{i}+\mathscr{O}(\delta),
$$

where $\hat{n}_{k}^{i}$ and $\hat{\phi}^{i}$ do not depend on space. We now integrate equations (1a) for $k=0,1$ and $(3)$ in the main text over the volume of the cell, apply the divergence theorem and implement the membrane conditions (4) and (5), to obtain the following problem at the 
leading order

$$
\begin{aligned}
& 3 \mathscr{P}+\mathscr{M}=0, \\
& \mathscr{J}_{1}^{i a}-2 \mathscr{P}+\mathscr{M}+\mathscr{J}_{1}^{i b}+\frac{2}{H} \int_{0}^{L} \mathscr{J}_{1}^{i g} d x=0 \\
& \mathscr{P}+\mathscr{J}_{1}^{i a}+\mathscr{J}_{1}^{i b}-\mathscr{J}_{2}^{i b}+\frac{2}{H} \int_{0}^{L}\left(\mathscr{J}_{1}^{i g}-\mathscr{J}_{2}^{i g}\right) d x=0,
\end{aligned}
$$

where we have to keep integrals only for the cleft variables, as the values in other regions are uniform. We note that all fluxes $\left(\mathscr{P}, \mathscr{M}, \mathscr{J}_{1}^{i a}, \mathscr{J}_{1}^{i b}, \mathscr{J}_{1}^{i g}, \mathscr{J}_{2}^{i b}\right.$ and $\left.\mathscr{J}_{2}^{i g}\right)$ are computed using the leading order, spatially constant variables in the cell. In the following we will not distinguish leading order variables from the exact variables; in other words we will write, for instance, $n_{k}^{i}$ rather than $\hat{n}_{k}^{i}$. In summary, this reduces the problem in the cell to a system of algebraic equations.

\section{Appendix B. Reduction of the ion transport model in the cleft to a set of ODEs}

In this section we derive the ODEs 14a-14c). We first redefine $y$ via $y \rightarrow y-$ $(h+H)$, so that the left wall of the cleft gap is at $y=0$ and the symmetry axes are at $710 y=h$ and $y=-H / 2$. It is convenient from now on to work in terms of dimensionless variables. Let $N$ denote a representative ion concentration, equivalent to $100 \mathrm{mM}$, corresponding to a physiological solution with molarity $300 \mathrm{mM}$. With $s \in\{a, b, i, g\}$ we non-dimensionalise all variables as follows

$$
x=L \bar{x}, \quad y=h \bar{y}, \quad n_{k}^{s}=N \bar{n}_{k}^{s}, \quad \phi^{s}=\frac{R T}{F} \bar{\phi}^{s}, \quad X=N \bar{X}, \quad u=U \bar{u}, \quad v=\delta U \bar{v},
$$

where the choice of fluid velocity scale $U \approx 60 \mu \mathrm{m} / \mathrm{s}$ is discussed in Appendix $\mathrm{D}$. In the cleft gap, electrodiffusion is governed by equations 1 a for $k=0,1$ and $(3$, which scale as follows

$$
\begin{aligned}
& \frac{\partial^{2} \bar{n}_{k}^{g}}{\partial \bar{x}^{2}}+z_{k} \frac{\partial}{\partial \bar{x}}\left(\bar{n}_{k}^{g} \frac{\partial \bar{\phi}^{g}}{\partial \bar{x}}\right)+\frac{1}{\delta^{2}}\left(\frac{\partial^{2} \bar{n}_{k}^{g}}{\partial \bar{y}^{2}}+z_{k} \frac{\partial}{\partial \bar{y}}\left(\bar{n}_{k}^{g} \frac{\partial \bar{\phi}^{g}}{\partial \bar{y}}\right)\right)-P e\left(\frac{\partial\left(\bar{u} \bar{n}_{k}^{g}\right)}{\partial \bar{x}}+\frac{\partial\left(\bar{v} \bar{n}_{k}^{g}\right)}{\partial \bar{y}}\right)=0,( \\
& \frac{\partial}{\partial \bar{x}}\left(\left[\sum_{k=0}^{2} \bar{n}_{k}^{g}\right] \frac{\partial \bar{\phi}^{g}}{\partial \bar{x}}\right)+\frac{1}{\delta^{2}} \frac{\partial}{\partial \bar{y}}\left(\left[\sum_{k=0}^{2} \bar{n}_{k}^{g}\right] \frac{\partial \bar{\phi}^{g}}{\partial \bar{y}}\right)=0
\end{aligned}
$$


where $P e$ is a Péclet number, defined as $P e=U L / D \approx 0.3$. Taking advantage of the small ratio $\delta$ of the cleft gap, we expand all variables in terms of $\delta$

$$
\bar{n}_{k}^{g}=\bar{n}_{k, 0}^{g}+\delta^{2} \bar{n}_{k, 1}^{g}+\mathscr{O}\left(\delta^{4}\right), \quad \bar{\phi}^{g}=\bar{\phi}_{0}^{g}+\delta^{2} \bar{\phi}_{1}^{g}+\mathscr{O}\left(\delta^{4}\right) .
$$

Introducing these expansions into equations $(\mathrm{B} .2)-(B .3)$ we obtain the following leading order problem (denoting for simplicity of the notation leading order variables as exact ones, e.g. using $\bar{n}_{k}^{g}, \bar{\phi}^{g}$ rather than $\bar{n}_{k, 0}^{g}$ and $\bar{\phi}_{0}^{g}$ )

$$
\frac{\partial}{\partial \bar{y}}\left(\left[\sum_{k=0}^{2} \bar{n}_{k}^{g}\right] \frac{\partial \bar{\phi}^{g}}{\partial \bar{y}}\right)=0, \quad \frac{\partial^{2} \bar{n}_{k}^{g}}{\partial \bar{y}^{2}}+z_{k} \frac{\partial}{\partial \bar{y}}\left(\bar{n}_{k}^{g} \frac{\partial \bar{\phi}^{g}}{\partial \bar{y}}\right)=0, \quad k=0,1 .
$$

Noting the symmetry condition at $\bar{y}=1$, where $\partial / \partial \bar{y}$ operating on any variable yields zero, we have for $k \in\{0,1\}$

$$
\frac{\partial \bar{n}_{k}^{g}}{\partial \bar{y}}+z_{k} \bar{n}_{k}^{g} \frac{\partial \bar{\phi}^{g}}{\partial \bar{y}}=0
$$

Hence

$$
\bar{n}_{k}^{g}=A_{k}(\bar{x}) \exp \left[-z_{k} \bar{\phi}^{g}\right]
$$

where $A_{0}$ and $A_{1}$ arise from the general form of the solution following integration. Noting that the valences for sodium and potassium $z_{0}=z_{1}=1$, and using electroneutrality in the gap, $\bar{n}_{2}^{g}=\bar{n}_{0}^{g}+\bar{n}_{1}^{g}$, we have

$$
2 \frac{\partial}{\partial \bar{y}}\left(\left[A_{0}(\bar{x})+A_{1}(\bar{x})\right] \exp \left[-\bar{\phi}^{g}\right] \frac{\partial \bar{\phi}^{g}}{\partial \bar{y}}\right)=0 .
$$

One integration and the symmetry condition gives

$$
\frac{\partial \bar{\phi}^{g}}{\partial \bar{y}}=0
$$

and hence the leading order bulk potential, and thus the concentrations, have no $\bar{y}$ dependence. 
With the 1 superscript denoting the first subleading term and integrating the firstorder correction to the concentration balance equation over $\bar{y}$, we have

$$
\int_{0}^{2}\left\{\frac{\partial^{2} \bar{n}^{g}}{\partial \bar{x}^{2}}+\frac{\partial}{\partial \bar{x}}\left(z_{k} \bar{n}_{k}^{g} \frac{\partial \bar{\phi}^{g}}{\partial \bar{x}}-P e\left(\bar{u} \bar{n}_{k}^{g}\right)\right)\right\} d \bar{y}+\left[\frac{\partial \bar{n}_{k, 1}^{g}}{\partial \bar{y}}+z_{k} \bar{n}_{k}^{g} \frac{\partial \bar{\phi}_{1}^{g}}{\partial \bar{y}}+z_{k} \bar{n}_{k, 1}^{g} \frac{\partial \bar{\phi}^{g}}{\partial \bar{y}}-P e\left(\bar{v} \bar{n}_{k}^{g}\right)\right]_{\bar{y}=0}^{\bar{y}=2}=0 .
$$

The contribution to the square bracket is determined by the boundary conditions at $\bar{y}=0$ and $\bar{y}=2$,

$$
\frac{\partial \bar{n}_{k, 1}^{g}}{\partial \bar{y}}+z_{k} \bar{n}_{k}^{g} \frac{\partial \bar{\phi}_{1}^{g}}{\partial \bar{y}}+z_{k} \bar{n}_{k, 1}^{g} \frac{\partial \bar{\phi}^{g}}{\partial \bar{y}}-P e \bar{v} \bar{n}_{k}^{g}= \pm \hat{P}_{k}^{b} z_{k}\left(\bar{\phi}^{i}-\bar{\phi}^{g}\right) \frac{\bar{n}_{k}^{i}-\bar{n}_{k}^{g} \exp \left[-z_{k}\left(\bar{\phi}^{i}-\bar{\phi}^{g}\right)\right]}{1-\exp \left[-z_{k}\left(\bar{\phi}^{i}-\bar{\phi}^{g}\right)\right]}
$$

where $\hat{P}_{k}^{b}=P_{k}^{b} \frac{L}{D \delta}$ and the right hand side sign is '+' for $\bar{y}=2$ and '-' for $\bar{y}=0$. As the membrane is impermeable to sodium, $P_{0}^{b}=0$. Non-zero values $\hat{P}_{k}^{b}$ have to be of order 1 for the above expression to be valid, and for the values reported in table 2 we find $\hat{P}_{1}^{b}=0.88, \hat{P}_{2}^{b}=0.05$. We denote the term on the right hand side of the expression B.11 as $\bar{J}_{k}^{i g}$, which represents the dimensionless lateral flux.

Recalling that concentrations and the potential under integral in equation $B .10$ are $\bar{y}$ independent, we rewrite the equation $(\overline{B .10})$, in the following way

$$
\frac{d^{2} \bar{n}_{k}^{g}}{d \bar{x}^{2}}+\frac{d}{d \bar{x}}\left(z_{k} \bar{n}_{k}^{g} \frac{d \bar{\phi}^{g}}{d \bar{x}}\right)-P e\left(\bar{q} \frac{d \bar{n}_{k}^{g}}{d \bar{x}}+\frac{\bar{n}_{k}^{g}}{2} \int_{0}^{2} \frac{\partial \bar{u}}{\partial \bar{x}} d \bar{y}\right)+\bar{J}_{k}^{i g}\left(\bar{n}_{k}^{g}, \bar{\phi}^{g}\right)=0, \quad k=0,1,
$$

where $\bar{q}=\frac{1}{2} \int_{0}^{2} \bar{u} d \bar{y}$ is dimensionless depth-averaged velocity. Using the continuity equation for the fluid $17 \mathrm{~b}$ ) to treat the term with integral, we obtain the following expression

$$
\frac{d^{2} \bar{n}_{k}^{g}}{d \bar{x}^{2}}+\frac{d}{d \bar{x}}\left(z_{k} \bar{n}_{k}^{g} \frac{d \bar{\phi}^{g}}{d \bar{x}}\right)-P e\left(\bar{q} \frac{d \bar{n}_{k}^{g}}{d \bar{x}}-\bar{Q}^{g} \bar{n}_{k}^{g}\right)+\bar{J}_{k}^{i g}\left(\bar{n}_{k}^{g}, \bar{\phi}^{g}\right)=0, \quad k=0,1,
$$

where $\bar{Q}^{g}$ is the water flux through the lateral membrane.

Using a similar procedure for $(\mathrm{B} .3)$ at order $\delta^{2}$ we obtain the following equation

$$
\frac{d}{d \bar{x}}\left(\left[\sum_{k=0}^{2} \bar{n}_{k}^{g}\right] \frac{d \bar{\phi}^{g}}{d \bar{x}}\right)+\overline{\mathscr{J}}_{1}^{i g}-\overline{\mathscr{J}}_{2}^{i g}=0 .
$$


The boundary conditions at the tight junction $(\bar{x}=1)(15)$ are rewritten as follows

$$
\begin{aligned}
& \frac{d \bar{n}_{k}^{g}}{d \bar{x}}+z_{k} \bar{n}_{k}^{g} \frac{d \bar{\phi}^{g}}{d \bar{x}}-P e \bar{Q}^{t j} \bar{n}_{k}^{g}=\overline{\mathscr{J}}_{k}^{a g}\left(\bar{n}_{k}^{g}, \bar{\phi}^{g}\right), \quad k=0,1 \\
& -2\left(\bar{n}_{0}^{g}+\bar{n}_{1}^{g}\right) \frac{d \bar{\phi}^{g}}{d \bar{x}}=\bar{J}_{1}^{a g}\left(A_{1}, \bar{\phi}^{g}\right),
\end{aligned}
$$

with Dirichlet conditions at $\bar{x}=0$

$$
\bar{n}_{0}^{g}=\bar{N}_{0}^{b}, \quad \bar{n}_{1}^{g}=\bar{N}_{1}^{b}, \quad \bar{\phi}^{g}=0 .
$$

We therefore have 3 coupled ODEs, B.13 - B.14, for 3 unknowns $\bar{n}_{0}^{g}, \bar{n}_{1}^{g}, \bar{\phi}^{g}$ with the boundary conditions $\mathrm{B} .15 \mathrm{a}, \mathrm{B} .15 \mathrm{~b}, \mathrm{~B} .16$.

\section{Appendix C. Derivation of the solution in the Debye layer}

In this section we describe the problem in the electrical double layer (EDL) and derive the solution. As reported in the main text, electroneutrality in the Debye layer breaks down and we have to solve the system (1a) and $1 \mathrm{~b}$.

We work in terms of dimensionless variables, as in Appendix B but introducing new spatial scales

$$
x=L X, \quad y=\lambda_{D} Y,
$$

${ }_{755}$ where $\lambda_{D}=\sqrt{\frac{\varepsilon R T}{F^{2} N}} \approx 1.8 \mathrm{~nm}$ is the Debye length. We also define the aspect ratio of the EDL, $\lambda=\lambda_{D} / L$. As in Appendix B, we assume that the left wall of the cleft is located at $y=0$.

Using $n_{k}^{d}, \phi^{d}$ to denote ion concentrations and electrical potential in the Debye layer of the cleft (denoted as $n_{k}^{E D L}$ and $\phi^{E D L}$ in the main text), we use a similar expansion as in the bulk of the cleft Appendix B but in terms of the EDL aspect ratio

$$
\phi^{d}=\bar{\phi}_{0}^{d}+\lambda^{2} \bar{\phi}_{1}^{d}+\mathscr{O}\left(\lambda^{4}\right), \quad \bar{n}_{k}^{d}=\bar{n}_{k, 0}^{d}+\lambda^{2} \bar{n}_{k, 1}^{d}+\mathscr{O}\left(\lambda^{4}\right), \quad k=0,1,2 .
$$

Rescaling equations $(1 \mathrm{a})$ and $(1 \mathrm{~b})$ and applying the above expansion at leading order (and again using the same notation for leading-order variables and exact ones), we have 


$$
\frac{\partial^{2} \bar{\phi}^{d}}{\partial Y^{2}}=-\left(\bar{n}_{0}^{d}+\bar{n}_{1}^{d}-\bar{n}_{2}^{d}\right), \quad \frac{\partial^{2} \bar{n}_{k}^{d}}{\partial Y^{2}}+\frac{\partial}{\partial Y}\left(z_{k} \bar{n}_{k}^{d} \frac{\partial \bar{\phi}^{d}}{\partial Y}\right)=0, \quad k=0,1,2
$$

Integrating the second expression and applying the leading-order boundary condition (4) we obtain

$$
\frac{\partial \bar{n}_{k}^{d}}{\partial Y}+z_{k} \bar{n}_{k}^{d} \frac{\partial \bar{\phi}^{d}}{\partial Y}=0
$$

Integrating the above equation and matching with the solution in the bulk, yields

$$
\bar{n}_{k}^{d}(X, Y)=\bar{n}_{k}^{g}(X, 0) \exp \left[-z_{k}\left(\bar{\phi}^{d}(X, Y)-\bar{\phi}^{g}(X, 0)\right)\right]
$$

where $\bar{n}_{k}^{g}(X, 0), \bar{\phi}^{g}(X, 0)$ are the inner limits of the bulk concentrations and potential. Noting that the outer variables have no $Y$ dependence (at leading order) and using the standard Huckel-Debye approximation [21] one has

$$
\frac{\partial^{2}}{\partial Y^{2}}\left(\bar{\phi}^{d}-\bar{\phi}^{g}\right)=\left(\sum_{k=0}^{2} \bar{n}_{k}^{g}\right)\left(\bar{\phi}^{d}-\bar{\phi}^{g}\right) .
$$

Hence, upon matching with the solution in the bulk, we have

$$
\bar{\phi}^{d}(X, Y)-\bar{\phi}^{g}=K(X) \exp \left[-\left(\sum_{k=0}^{2} \bar{n}_{k}^{g}\right)^{1 / 2} Y\right]
$$

770 where $K(X)$ is given by $\bar{\phi}^{d}(X, 0)-\bar{\phi}^{g}$. Note that $\bar{\phi}^{d}(X, 0)$ is still undetermined and, to find it, we require the boundary condition for the potential at $Y=0$

$$
\left.\frac{\partial \bar{\phi}^{d}}{\partial Y}\right|_{Y=0}=\bar{\sigma}
$$

where $\bar{\sigma}$ is the non-dimensionalisation of

$$
\sigma=C_{m}\left(\phi^{g}-\phi^{i}\right)-\sigma_{0}
$$

and it takes into account the surface charge due to the presence of proteins via $\sigma_{0}$ and 
has a term which is proportional to the jump of the limits of the bulk potentials as they approach the membrane with the membrane capacitance $C_{m}$. The scale for the surface charge density is FNLג (for the meaning of the symbols please refer to Table 2). The condition at $Y=0$ is therefore given by

$$
\frac{\partial \bar{\phi}^{d}}{\partial Y}=\chi\left(\bar{\phi}^{g}-\bar{\phi}^{i}\right)-\bar{\sigma}_{0}
$$

where $\chi=\frac{C_{m} L}{\varepsilon} \lambda \approx 0.02$.

Applying this condition we obtain the inner solution $\bar{\phi}^{d}$. We note that by construction, the inner solution coincides with the composite solution, which we denote with $\Phi$, and it reads

$$
\bar{\Phi}(X, Y)=\bar{\phi}^{g}-\frac{\chi\left(\bar{\phi}^{g}-\bar{\phi}^{i}\right)-\bar{\sigma}_{0}}{\left(\sum_{k=0}^{2} \bar{n}_{k}^{g}\right)^{1 / 2}} \exp \left[-\left(\sum_{k=0}^{2} \bar{n}_{k}^{g}\right)^{1 / 2} Y\right]
$$

\section{Appendix D. Derivation of the expression for the slip velocity}

We start with the equations (17) for the fluid flow in the EDL.

For convenience, we work in terms of dimensionless variables and introduce the following scales

$$
x=L X, \quad y=\lambda_{D} Y, \quad u=U \bar{u}, \quad v=\delta U \bar{v}, \quad \Phi=\frac{R T}{F} \bar{\Phi}, \quad p=P_{0} \bar{p},
$$

where $\lambda_{D}$ is the Debye length, and $U=\frac{\varepsilon}{L \mu}\left(\frac{R T}{F}\right)^{2}=60 \mu \mathrm{m} / \mathrm{s}$. The scale for the pressure is chosen so that the pressure gradient balances with the dominant viscous term, to obtain $P_{0}=L \mu U / \lambda_{D}^{2}$. Since the aspect ratio of the Debye layer $\lambda=\lambda_{D} / L$ is small we use lubrication theory to approximate the Navier Stokes equations. In particular we drop terms of order $\lambda^{2}$ or smaller to obtain the following system of dimensionless equations

$$
\begin{aligned}
& -\frac{\partial \bar{p}}{\partial X}+\frac{\partial^{2} \bar{u}}{\partial X^{2}}+\frac{\partial^{2} \bar{\Phi}}{\partial Y^{2}} \frac{\partial \bar{\Phi}}{\partial X}=0, \\
& -\frac{\partial \bar{p}}{\partial Y}+\frac{\partial^{2} \bar{\Phi}}{\partial Y^{2}} \frac{\partial \bar{\Phi}}{\partial Y}=0 .
\end{aligned}
$$


Using [C.5] it is convenient to redefine the pressure, so that $p^{\prime}=\bar{p}-\frac{C(X)}{2}\left(\bar{\Phi}-\bar{\phi}^{g}\right)^{2}$, where $C(X)=\sum_{k=0}^{2} \bar{n}_{k}^{g}(X)$ and $\bar{\phi}^{g}$ is the potential in the bulk. With this redefined pressure the above equations take the form

$$
\begin{aligned}
& -\frac{\partial p^{\prime}}{\partial X}+\frac{\partial^{2} \bar{u}}{\partial Y^{2}}+\frac{\partial^{2} \bar{\Phi}}{\partial Y^{2}} \frac{\partial \bar{\phi}^{g}}{\partial X}-C^{\prime}(X) \frac{(\bar{\sigma})^{2}}{2 C(X)} \exp [-2 \sqrt{C(X)} Y]=0, \\
& \frac{\partial p^{\prime}}{\partial Y}=0
\end{aligned}
$$

795 twice with respect to $Y$ and apply the no slip boundary condition at the wall $Y=0$ to obtain

$$
\begin{array}{r}
\bar{u}^{E D L}=\frac{d p^{\prime}}{d X} \frac{Y^{2}}{2}+c_{1}(X) Y+\left(\frac{\bar{\sigma}(\exp [-\sqrt{C(X)} Y]-1)}{\sqrt{C(X)}}\right) \frac{\partial \bar{\phi}^{g}}{\partial X}+ \\
+C^{\prime}(X) \frac{(\bar{\sigma})^{2}}{8 C^{2}(X)}(\exp [-2 \sqrt{C(X)} Y]-1)
\end{array}
$$

and the constant $c_{1}(X)$ can be determined by matching this solution (inner) with that in the bulk of the cleft gap (outer). In the above expression, we have added a superscript $E D L$ to denote the velocity in this region to distinguish it from the solution in the bulk of the cleft.

In the bulk of cleft (outer region) the fluid flow is governed by Stokes equation

$$
-\nabla p+\mu \nabla^{2} \mathbf{u}=0
$$

A similar analysis as for the inner region can be applied also in this case. The scaling will differ from the one above only for the variables $y=h \bar{y}$ and $p=L \mu U / h^{2} \bar{p}$, and the spatial aspect ratio is $\delta=h / L$.

Rescaling the equations and dropping terms of order $\delta^{2}$ or smaller we obtain the 
following equations

$$
\begin{aligned}
& -\frac{\partial \bar{p}}{\partial \bar{x}}+\frac{\partial^{2} \bar{u}}{\partial \bar{x}^{2}}=0, \\
& -\frac{\partial \bar{p}}{\partial \bar{y}}=0 .
\end{aligned}
$$

Hence

$$
\bar{u}^{b u l k}=\frac{\partial \bar{p}}{\partial \bar{x}} \frac{\bar{y}^{2}}{2}+d_{1}(\bar{x}) \bar{y}+d_{2}(\bar{x})
$$

where we added the superscript bulk to distinguish this velocity from that of the inner region (D.4). Applying the symmetry condition in $y$-direction we get $d_{1}(\bar{x})=0$. To determine the other constant, we perform matching with the inner region (and recall that in the bulk $\bar{p}=p^{\prime}$ )

$$
d_{2}(\bar{x})=-\frac{\bar{\sigma}}{\sqrt{C(\bar{x})}} \frac{\partial \bar{\phi}^{g}}{\partial \bar{x}}-C^{\prime}(\bar{x}) \frac{(\bar{\sigma})^{2}}{8 C^{2}(\bar{x})},
$$

and $c_{1}(\bar{x})=0$. The slip velocity is thus defined as $u_{s l i p}=d_{2}(\bar{x})$.

\section{Appendix E. Solution of the fluid flow in the cleft}

We now work in the bulk of the cleft and shift the $\bar{y}$-axis into the centre of the cleft, so that the cleft spans from $\bar{y}=-1$ to $\bar{y}=1$ and the symmetry axis is at $\bar{y}=0$. In the bulk of the cleft the velocity profile is then given by

$$
\bar{u}=\frac{1}{2} \frac{\partial \bar{p}}{\partial \bar{x}}\left(\bar{y}^{2}-1\right)+u_{\text {slip }}
$$

The equation for the pressure is obtained by integrating the continuity equation along the thickness of the cleft with the boundary conditions $\bar{v}(\bar{y}= \pm 1)= \pm \bar{Q}^{g}(\bar{x})$, where $\bar{Q}^{g}=Q^{g} / \delta U=k_{m} / \delta U\left[R T\left(\sum_{k=0}^{2}\left(n_{k}^{i}-n_{k}^{g}(x)\right)+X\right)+p(x)\right]$ is the dimensionless lateral flux per unit area. The equation for the pressure $\bar{p}$ then becomes

$$
-\frac{1}{3} \frac{d^{2} \bar{p}}{d \bar{x}^{2}}+\frac{d u_{\text {slip }}}{d \bar{x}}=-\bar{Q}^{g}(\bar{x}),
$$


subject to the boundary conditions

$$
\begin{aligned}
& \bar{p}(\bar{x})=0, \\
& -\left.\frac{1}{3} \frac{d \bar{p}}{d \bar{x}}\right|_{\bar{x}=1}+u_{\text {slip }}(1)=\bar{Q}^{t j},
\end{aligned}
$$

where $\bar{Q}^{t j}=Q^{t j} / U$ is the dimensionless flux through the tight junction and is given by the expression $Q^{t j}=k_{t j}\left(R T \sum_{k=0}^{1}\left(n_{k}^{a}-n_{k}^{g}(L)\right)+p(L)\right)$.

\section{Competing interests}

None of the authors has competing interests.

\section{Funding}

The work has been supported by the Macular Society and the UK Fluids Network, funded by EPSRC grant EP/NO32861/1.

\section{Acknowledgements}

The authors thank Prof. Federica Grillo for drawing Figure 1 . We thank the anonymous reviewers for providing very useful suggestions, which contributed significantly to improving the paper.

\section{References}

[1] H. Kolb, Simple anatomy of the retina by helga kolb (2011).

URL http://webvision.med.utah.edu/book/part-i-foundations/ simple-anatomy-of-the-retina/

[2] R. K. Sharma, B. Ehinger, Development and structure of the retina, Adler's Physiology of the Eye: Clinical Applications 10 (2003) 319-347.

840 [3] M. La Cour, The retinal pigment epithelium, Adler's Physiology of the Eye: Clinical Applications 10 (2003) 348-357. 
[4] R. Gallemore, B. Hughes, S. Miller, Transport mechanisms in the retinal pigment epithelium, in: M. Marmor, T. Wolfensberger (Eds.), The Retinal Pigment Epithelium, Oxford University Press New York, 1998, pp. 103-134.

[5] M. Dornonville de la Cour, Ion transport in the retinal pigment epithelium. a study with double barrelled ion-selective microelectrodes., Acta. Ophthalmol. Suppl. (209) (1993) 1.

[6] N. Reichhart, O. Strauß, Ion channels and transporters of the retinal pigment epithelium, Exp. Eye. Res. 126 (2014) 27-37. doi:10.1016/j.exer.2014.05. 005.

[ [7] K. R. Spring, Mechanism of fluid transport by epithelia, Compr. Physiol doi: 10.1002/cphy.cp060405.

[8] O. Strauss, The retinal pigment epithelium

[9] M. Marmor, Control of subretinal fluid: experimental and clinical studies, Eye 4 (2) (1990) 340-344. doi:10.1038/eye.1990.46

[10] S. Hamann, Molecular mechanisms of water transport in the eye, Int. Rev. Cytol. 215 (2002) 395-431. doi:10.1016/S0074-7696(02) 15016-9.

[11] A. M. Weinstein, J. L. Stephenson, Models of coupled salt and water transport across leaky epithelia, The Journal of membrane biology 60 (1) (1981) 1-20.

[12] J. Fischbarg, Fluid transport across leaky epithelia: central role of the tight junction and supporting role of aquaporins, Physiol Rev 90 (4) (2010) 1271-1290. doi:10.1152/physrev.00025.2009

[13] J. Fischbarg, On the mechanism of fluid transport across corneal endothelium and epithelia in general, Journal of Experimental Zoology Part A: Ecological Genetics and Physiology 300 (1) (2003) 30-40. doi:10.1002/jez.a.10306. 
[14] A. Hill, Fluid transport: a guide for the perplexed, J. Membr. Biol. 223 (1) (2008) 1-11. doi:10.1007/s00232-007-9085-1.

[15] J. M. Diamond, W. H. Bossert, Standing-gradient osmotic flow, J. Gen. Physiol. 50 (8) (1967) 2061-2083.

[16] S. G. Schultz, The role of paracellular pathways in isotonic fluid transport, Yale J. Biol. Med. 50 (2) (1977) 99.

[17] B. Shachar-Hill, A. E. Hill, Paracellular fluid transport by epithelia, Int. Rev. Cytol. 215 (2002) 319-350. doi:10.1016/S0074-7696(02) 15014-5

[18] A. Hill, B. Shachar-Hill, Y. Shachar-Hill, What are aquaporins for?, J. Membr. Biol. 197 (1) (2004) 1-32. doi:10.1007/s00232-003-0639-6

[19] J. Sanchez, V. Cacace, C. Kusnier, R. Nelson, A. Rubashkin, P. Iserovich, J. Fischbarg, Net fluorescein flux across corneal endothelium strongly suggests fluid transport is due to electro-osmosis, J. Membr. Biol. 249 (4) (2016) 469-473.

[20] J. Sanchez, Y. Li, A. Rubashkin, P. Iserovich, Q. Wen, J. Ruberti, R. Smith, D. Rittenband, K. Kuang, F. Diecke, et al., Evidence for a central role for electroosmosis in fluid transport by corneal endothelium, J. Membr. Biol. 187 (1) (2002) $37-50$

[21] R. F. Probstein, Physicochemical hydrodynamics: an introduction, John Wiley \& Sons, 2005.

[22] S. S. Miller, R. H. Steinberg, B. Oakley II, The electrogenic sodium pump of the frog retinal pigment epithelium, J. Membr. Biol. 44 (3-4) (1978) 259-279.

[23] J. Adijanto, T. Banzon, S. Jalickee, N. S. Wang, S. S. Miller, Co2-induced ion and fluid transport in human retinal pigment epithelium, J. Gen. Physiol. 133 (6) (2009) 603-622. doi:10.1085/jgp. 200810169

[24] S. S. Miller, J. L. Edelman, Active ion transport pathways in the bovine retinal pigment epithelium., J. Physiol. (Lond) 424 (1990) 283. 
[25] S. Wimmers, M. O. Karl, O. Strauss, Ion channels in the rpe, Progress in retinal and eye research 26 (3) (2007) 263-301.

[26] S. Peng, R. A. Adelman, L. J. Rizzolo, Minimal effects of vegf and anti-vegf drugs on the permeability or selectivity of rpe tight junctions, Invest. Ophthalmol. Vis. Sci. 51 (6) (2010) 3216-3225. doi:10.1167/iovs.09-4162

[27] M. Marmor, Structure, function, and disease of the retinal pigment epithelium, The Retinal Pigment Epithelium (1998) 3-12.

[28] N. Gavish, K. Promislow, Dependence of the dielectric constant of electrolyte solutions on ionic concentration: A microfield approach, Phys. Rev. E 94 (1) (2016) 012611.

[29] G. G. Matthews, Cellular physiology of nerve and muscle, John Wiley \& Sons, 2009.

[30] S. McLaughlin, R. T. Mathias, Electro-osmosis and the reabsorption of fluid in renal proximal tubules., J. Gen. Physiol. 85 (5) (1985) 699-728.

[31] B. A. Hughes, S. S. Miller, T. E. Machen, Effects of cyclic amp on fluid absorption and ion transport across frog retinal pigment epithelium. measurements in the open-circuit state., J. Gen. Physiol. 83 (6) (1984) 875-899.

[32] G. Shi, A. Maminishkis, T. Banzon, S. Jalickee, R. Li, J. Hammer, S. S. Miller, Control of chemokine gradients by the retinal pigment epithelium, Invest. Ophthalmol. Vis. Sci. 49 (10) (2008) 4620-4630. doi:10.1167/iovs.08-1816.

[33] R. Mathias, H. Wang, Local osmosis and isotonic transport, J. Membr. Biol. 208 (1) (2005) 39-53. doi : 10.1007/s00232-005-0817-9

[34] T. Weiss, Cellular Biophysics I. Transport., MIT Press, Cambridge, Massachusetts, 1996.

[35] T. Weiss, Cellular Biophysics II. Electrical Properties., MIT Press, Cambridge, Massachusetts, 1996. 
[36] Y. Mori, C. Peskin, A numerical method for cellular electrophysiology based on the electrodiffusion equations with internal boundary conditions at membranes,

1 Comm. App. Math. and Comp. Sci. 4 (2009) 85-134. doi:10.2140/camcos. 2009.4 .85

[37] Y. Mori, J. Jerome, C. Peskin, A three-dimensional model of cellular electrical activity, Bull. Inst. Math. Acad. Sinica 2 (2007) 367-390.

[38] H. Li, D. N. Sheppard, M. J. Hug, Transepithelial electrical measurements with the ussing chamber, Journal of Cystic Fibrosis 3 (2004) 123-126.

[39] J. P. Keener, J. Sneyd, Mathematical physiology, Vol. 1, Springer, 2009.

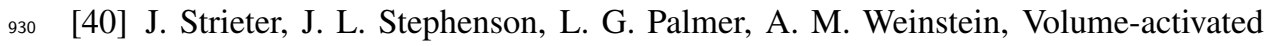
chloride permeability can mediate cell volume regulation in a mathematical model of a tight epithelium., J. Gen. Physiol. 96 (2) (1990) 319-344.

[41] Comsol multiphysics $(R, \quad$ https://www.comsol.com/ comsol-multiphysics, accessed: 2017-09-04.

[42] T. M. Squires, M. Z. Bazant, Induced-charge electro-osmosis, Journal of Fluid Mechanics 509 (2004) 217-252.

[43] E. Yariv, An asymptotic derivation of the thin-debye-layer limit for electrokinetic phenomena, Chemical Engineering Communications 197 (1) (2009) 3-17.

[44] S. Kusaka, A. Inanobe, A. Fujita, Y. Makino, M. Tanemoto, K. Matsushita, Y. Tano, Y. Kurachi, Functional kir7. 1 channels localized at the root of apical processes in rat retinal pigment epithelium, J. Physiol. (Lond) 531 (1) (2001) 2736.

[45] M. La Cour, Cl- transport in frog retinal pigment epithelium, Exp. Eye. Res. 54 (6) (1992) 921-931. doi:10.1016/0014-4835(92)90156-M.

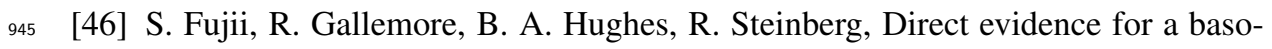
lateral membrane cl-conductance in toad retinal pigment epithelium, American Journal of Physiology-Cell Physiology 262 (2) (1992) C374-C383. 
[47] S. Bialek, S. S. Miller, K+ and cl-transport mechanisms in bovine pigment epithelium that could modulate subretinal space volume and composition., J. Physiol. (Lond) 475 (3) (1994) 401-417.

[48] V. Lew, H. Ferreira, T. Moura, The behaviour of transporting epithelial cells. i. computer analysis of a basic model, Proc. R. Soc. Lond.-B Biol. Sci. 206 (1162) (1979) 53-83.

[49] A. Rubashkin, P. Iserovich, J. Hernandez, J. Fischbarg, Epithelial fluid transport: protruding macromolecules and space charges can bring about electro-osmotic

a coupling at the tight junctions, J. Membr. Biol. 208 (3) (2006) 251-263. doi : $10.1007 / \mathrm{s} 00232-005-0831-\mathrm{y}$.

[50] W. D. Stamer, D. Bok, J. Hu, G. J. Jaffe, B. S. McKay, Aquaporin-1 channels in human retinal pigment epithelium: role in transepithelial water movement, Invest. Ophthalmol. Vis. Sci. 44 (6) (2003) 2803-2808. doi : 10.1167/iovs.03-0001.

[51] K. Juuti-Uusitalo, C. Delporte, F. Grégoire, J. Perret, H. Huhtala, V. Savolainen, S. Nymark, J. Hyttinen, H. Uusitalo, F. Willermain, et al., Aquaporin expression and function in human pluripotent stem cell-derived retinal pigmented epithelial cells, Investigative ophthalmology \& visual science 54 (5) (2013) 3510-3519.

[52] R. P. Gallemore, R. H. Steinberg, Effects of dids on the chick retinal pigment epithelium. i. membrane potentials, apparent resistances, and mechanisms, Journal of Neuroscience 9 (6) (1989) 1968-1976.

[53] D. Frambach, J. Valentine, J. Weiter, Furosemide-sensitive cl transport in bovine retinal pigment epithelium., Invest. Ophthalmol. Vis. Sci. 30 (10) (1989) 22712274.

[54] R. Quinn, S. Miller, Ion transport mechanisms in native human retinal pigment epithelium., Invest. Ophthalmol. Vis. Sci. 33 (13) (1992) 3513-3527.

[55] J. M. Anderson, C. M. Van Itallie, Physiology and function of the tight junca tion, CSH. Perspect. Biol. 1 (2) (2009) a002584. doi:10.1101/cshperspect. a002584 
[56] R. T. Mathias, Epithelial water transport in a balanced gradient system, Biophysical journal 47 (6) (1985) 823-836.

[57] S. S. Miller, R. H. Steinberg, Passive ionic properties of frog retinal pigment epithelium, J. Membr. Biol. 36 (1) (1977) 337-372.

[58] J. Fischbarg, F. Diecke, A mathematical model of electrolyte and fluid transport

口 across corneal endothelium, J. Membr. Biol. 203 (1) (2005) 41-56. doi:10. 1007/s00232-004-0730-7.

[59] J. Fischbarg, F. Diecke, P. Iserovich, A. Rubashkin, The role of the tight junction in paracellular fluid transport across corneal endothelium. electro-osmosis as a driving force, J. Membr. Biol. 210 (2) (2006) 117-130. doi:10.1007/ s00232-005-0850-8

[60] A. M. Weinstein, E. D. Sontag, Modeling proximal tubule cell homeostasis: tracking changes in luminal flow, Bulletin of mathematical biology 71 (6) (2009) $1285-1322$.

990 [61] A. Hill, Solute-solvent coupling in epithelia: a critical examination of the standing-gradient osmotic flow theory, Proc. R. Soc. Lond.-B Biol. Sci. 190 (1098) (1975) 99-114.

[62] L. J. Rizzolo, S. Peng, Y. Luo, W. Xiao, Integration of tight junctions and claudins with the barrier functions of the retinal pigment epithelium, Prog. Retin. Eye Res. 30 (5) (2011) 296-323. 